\title{
Room-Temperature, Base-Mediated Selective Synthesis of 2-(Arylamino)ethanols and 2-Aryloxyethanols
}

\author{
Rahul B. Sonawane \\ Swapnali R. Sonawane \\ Nishant K. Rasal \\ Sangeeta V. Jagtap* (D)
}

Department of Chemistry, Baburaoji Cholap College, Sangvi, Pune 411027, India (Affiliated to Savitribai Phule Pune University, Pune, India)

sangeetajagtap@rediffmail.com
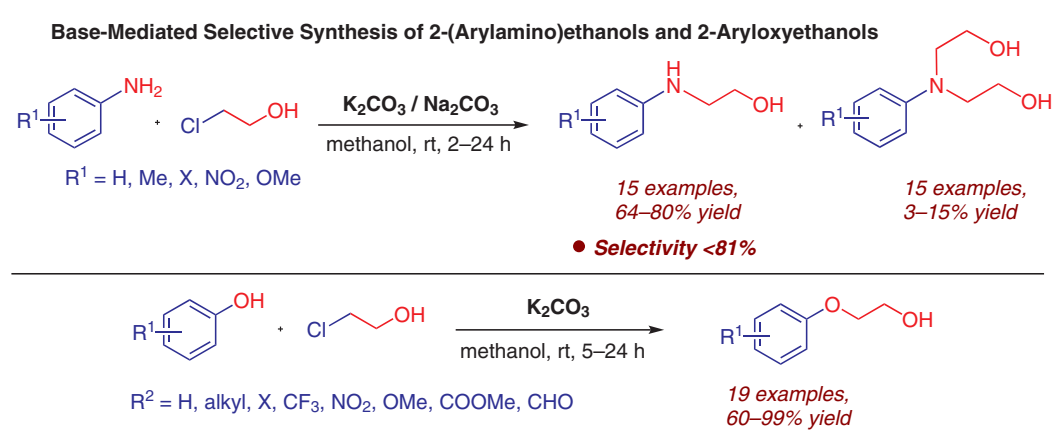

- Mild conditions - Chemoselective - Broad functional group tolerance - Ticlopidine, Vildagliptin, Quetiapine and Gemfibrozil drug synthesis

\begin{abstract}
Received: 22.09.2019
Accepted after revision: 31.10 .2019

Published online: 18.11 .2019

DOI: 10.1055/s-0039-1690334; Art ID: so-2019-d0026-op

License terms: CCO

(c) 2019. The Author(s). This is an open access article published by Thieme under the terms of the Creative Commons Attribution-NonDerivative-NonCommercial-License, permitting copying and reproduction so long as the original work is given appropriate credit. Contents may not be used for commercial purposes or adapted, remixed, transformed or built upon. (https://creativecommons.org/licenses/by-nc-nd/4.0/)

Abstract A simple and efficient protocol for base-mediated selective synthesis of 2-(arylamino)ethanols from primary aromatic amines and 2-aryloxyethanols from phenols, promoted by $\mathrm{K}_{2} \mathrm{CO}_{3}$ has been achieved under mild conditions. Even in presence of excess alkyl halide, selective mono- $\mathrm{N}$-alkylation has been achieved. Tolerance of a variety of functional groups is demonstrated by 15 examples of selective $\mathrm{N}$-alkylation of aromatic amines and 19 examples of O-alkylation of phenols. The efficacy of the protocol is demonstrated by the formal synthesis of Ticlopidine $^{\circledR}$, Vildagliptin $^{\circledR}$, Quetiapine ${ }^{\circledR}$, and Gemfibrozil ${ }^{\circledR}$.
\end{abstract}

Key words selective $\mathrm{N}$-alkylation, 2-(arylamino)ethanols, amines, 2aryloxyethanols, phenols, $\mathrm{K}_{2} \mathrm{CO}_{3}$ promoted, $\mathrm{Na}_{2} \mathrm{CO}_{3}$ controlled

$\mathrm{N}$-Alkylation of amines is central to the synthesis of synthetic intermediates, ${ }^{1}$ fine chemicals, ${ }^{2}$ pharmaceuticals, ${ }^{3}$ agrochemicals, ${ }^{4}$ dyes, ${ }^{5}$ rubbers, ${ }^{6}$ and polymers. ${ }^{7}$ Likewise, 0 -alkyl phenols are applied in paints, varnishes, printing inks, foaming agents, synthetic resins, and perfumes. ${ }^{8}$ Moreover, many important drug molecules have $\mathrm{N}$ alkyl moieties, including Metronidazole ${ }^{\circledR}$, Fluphenazine ${ }^{\circledR}$, Quetiapine $^{\circledR}$, Vildagliptin ${ }^{\circledR}$, Ticlopidine ${ }^{\circledR}$, and Ditazole ${ }^{\circledR}($ Figure 1).

Under standard conditions, amines commonly undergo over-alkylation, ${ }^{9}$ which leads to mixtures of secondary and tertiary amines and quaternary ammonium salts instead of the desired mono-N-alkylation products. Methods for preparing $\mathrm{N}$-alkyl amines include direct $\mathrm{N}$-alkylation of primary amines with alkyl halides, ${ }^{10}$ reduction of amides, ${ }^{11}$ re-
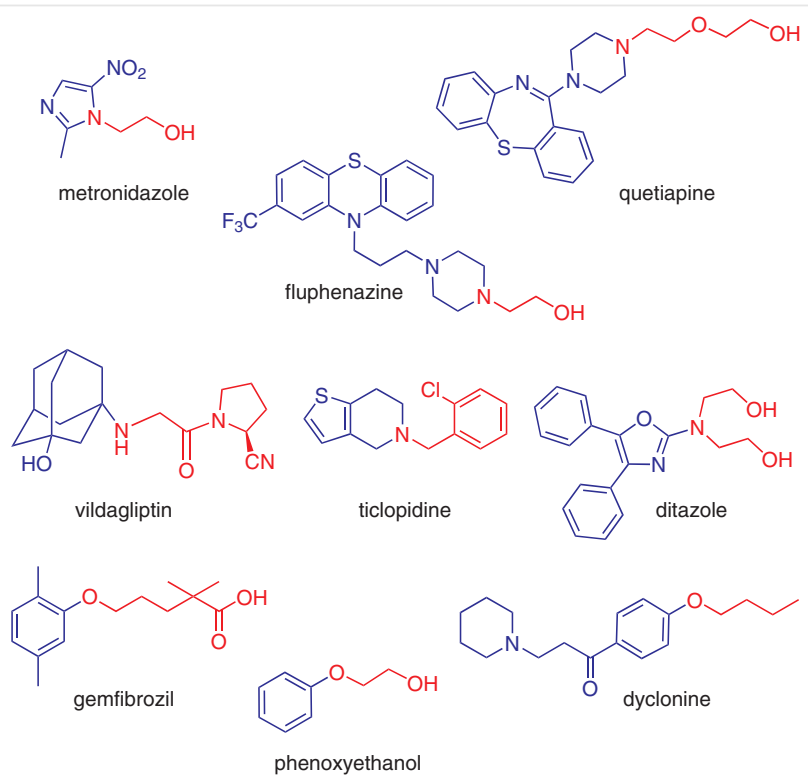

Figure 1 Drug molecules having $\mathrm{N}$-alkyl and $\mathrm{O}$-alkyl moieties

ductive amination of aldehydes with primary amines in the presence of reducing agents, ${ }^{12} \mathrm{~N}$-dealkylation of tertiary amines, ${ }^{13} \mathrm{C}-\mathrm{N}$ bond-coupling reactions, ${ }^{14}$ and transitionmetal-catalyzed direct alkylation of amines with alcohols by the borrowing hydrogen strategy. ${ }^{3,15} \mathrm{~N}$-Alkylation of amines can be achieved by using various alkyl sources such as alkyl halides, ${ }^{16}$ alcohols, ${ }^{3,17}$ dimethyl carbonate, ${ }^{17,18}$ ethylene glycol, ${ }^{19}$ epoxides, ${ }^{20}$ 2-chloroethanol, ${ }^{21} \mathrm{CO}_{2},{ }^{22}$ and $\mathrm{ZnEt}_{2}{ }^{23}$ In addition, various inorganic bases have been employed, such as $\mathrm{K}_{2} \mathrm{CO}_{3},{ }^{24} \mathrm{NaHCO},{ }_{3},{ }^{25} \mathrm{NaH},{ }^{26} \mathrm{CsOH} \cdot \mathrm{H}_{2} \mathrm{O},{ }^{27}$ and $\mathrm{CS}_{2} \mathrm{CO}_{3}{ }^{28}$ In addition methods have been described using ionic liquids ${ }^{29}$ and $\mathrm{N}$-sulfonamides $\mathrm{s}^{8,30}$ with alkyl halides. 
Recently, N-alkylation of anilines with alcohols has been performed efficiently by using $\mathrm{Mn},{ }^{31} \mathrm{Ni},{ }^{32}$ and $\mathrm{Ru}^{33}$ metal catalysts and alkyl halides on silica. ${ }^{34}$

Similarly, 0-alkylation of phenols is commonly carried out by Williamson's ether synthesis ${ }^{35}$ and $\mathrm{C}-\mathrm{O}$ bondcoupling reactions ${ }^{36}$ with alkyl halides. For O-alkylation of phenols the alkylating agents include alkyl halides, ${ }^{37}$ alcohols, ${ }^{38}$ dimethyl carbonate, ${ }^{39}$ allylic carboxylates, ${ }^{40}$ ethylene carbonate, ${ }^{41}$ methyl formate, ${ }^{35 \mathrm{~b}} 2$-chloroethanol, ${ }^{42}$ and epoxides. ${ }^{43}$ Equally, various inorganic bases have been employed, including $\mathrm{NaOH},{ }^{44} \mathrm{NaH},{ }^{45} \mathrm{~K}_{2} \mathrm{CO}_{3},{ }^{24 a, 42,43 \mathrm{~b}, 46}$ and $\mathrm{Cs}_{2} \mathrm{CO}_{3},{ }^{47}$ and ionic liquids have also been used. ${ }^{48}$

Nevertheless, the development of new methodologies for selective mono-N-alkylation and $\mathrm{O}$-alkylation protocols continues to be a major challenge. Therefore, the development of effective methods for such conversions continues to be a focus of attention. In particular, most traditional methods for the synthesis of aryl ethers require harsh conditions, such as strong bases and high temperatures, ${ }^{35 a, 49}$ and are incompatible with a range of functional groups..

Herein, we report a selective and simple protocol to synthesize 2-(arylamino)ethanols from a range of primary aromatic amines and 2-aryloxyethanols from several phenols with 2-chloroethanol, promoted by $\mathrm{K}_{2} \mathrm{CO}_{3}$ in methanol (Table 1 and Schemes 1-2). The corresponding mono-N-alkylated 2-(phenylamino)ethanol products are isolated with high selectivity (81-96\%) and moderate to excellent yields (64-80\%). Similarly, O-alkylated 2-phenoxyethanol products can also be synthesized with moderate to excellent yields (60-99\%). A wide range of functional groups is tolerated due to the mild reaction conditions for both $\mathrm{N}$ - and $\mathrm{O}-$ alkylation.

In preliminary reactions, 4-methylaniline (1c) was treated with 2-chloroethanol (2a) in the presence of an organic base (1 equiv) such as triethylamine or $\mathrm{N}, \mathrm{N}$-diisopropylethylamine (DIPEA) with methanol as solvent at ambient temperature, when $27-34 \%$ yield of the desired mono-N-alkylated product 3c and 35-38\% of di-N-alkylated product 4c were isolated (Table 1, entries 1 and 2). By using inorganic bases such as $\mathrm{Na}_{2} \mathrm{CO}_{3}, \mathrm{NaHCO}_{3}$ and $\mathrm{KHCO}_{3}$, the reactions were unsuccessful, with negligible yields of $\mathbf{3 c}$ and $\mathbf{4 c}$ being observed (entries 3-5). Other bases, such as $\mathrm{K}_{2} \mathrm{CO}_{3}, \mathrm{Cs}_{2} \mathrm{CO}_{3}$, $\mathrm{K}_{3} \mathrm{PO}_{4}, \mathrm{NaOH}, \mathrm{KOH}$, and $\mathrm{NaOMe}$, were found to be effective, but did not selectively furnish mono-N-alkylated product 3c (entries 6-11). While some selectivity was observed towards mono-N-alkylated product $3 \mathrm{c}$ with $\mathrm{K}_{2} \mathrm{CO}_{3}$ and, being aware of the relative solubility of $\mathrm{K}_{2} \mathrm{CO}_{3}$ in methanol, ${ }^{50}$ to control the over-alkylation, the reaction was carried out without any solvent, but satisfactory results were still not observed (entry 12).

After a revaluation of all the trials, it was clear that 1 equivalent of $\mathrm{Na}_{2} \mathrm{CO}_{3}, \mathrm{NaHCO}_{3}$ and $\mathrm{KHCO}_{3}$ showed low conversions but the selectivity was high; whereas 1 equivalent of $\mathrm{K}_{2} \mathrm{CO}_{3}$ promoted the reaction with a $3 \mathbf{c} / \mathbf{4} \mathbf{c}$ selectivity up to 58:42. Hence, the decision was made to use a mixture of 1 equivalent of $\mathrm{K}_{2} \mathrm{CO}_{3}$ and 1-3 equivalents of $\mathrm{Na}_{2} \mathrm{CO}_{3}$; whereupon, dramatic improvements in both selectivity and conversion were obtained (entries 13-15). Among these conditions, 3 equiv of $\mathrm{Na}_{2} \mathrm{CO}_{3}$ was most effective; under these conditions improvements in both selectivity $(\mathbf{3 c} / \mathbf{4 c}$, $84: 16)$ and conversion (91\%) were obtained, with $76 \%$ isolated yield of desired product $3 \mathbf{c}$ and only $15 \%$ yield of product 4c (entry 15). To examine the effect of $\mathrm{Na}_{2} \mathrm{CO}_{3}$ on this conversion, the reaction was carried out by replacing the $\mathrm{Na}_{2} \mathrm{CO}_{3}$ with $\mathrm{NaHCO}_{3}$ and $\mathrm{KHCO}_{3}$; in these cases slight decreases in selectivity and conversion were observed (entries 16 and 17). Changing $\mathrm{K}_{2} \mathrm{CO}_{3}$ with triethylamine, $\mathrm{Cs}_{2} \mathrm{CO}_{3}$ and $\mathrm{K}_{3} \mathrm{PO}_{4}$ led to a decrease in selectivity (entries 18-20).

Subsequently, various solvents such as acetonitrile, acetone, dichloromethane, THF, toluene, 1,4-dioxane, DMF, DMSO and NMP were screened, but none were successful (Table 1, entries 21-29), indicating that methanol is the most efficient solvent for this reaction. To examine the effect of alcoholic solvents on conversion and selectivity, reactions were carried out with ethanol and isopropanol, but decreases in selectivity were observed (entries 30-31). To examine the effect of the concentration of 2-chloroethanol on the reaction, experiments were performed using 1 and 2 equivalent of 2-chloroethanol $\mathbf{2 a}$, whereupon a notable decrease in both selectivity and conversion was observed (entries 32 and 33). At the end of this study, one experiment was performed by varying the temperature from ambient temperature up to $40{ }^{\circ} \mathrm{C}$ and then at reflux, but decreased selectivity at the higher temperature was observed (entry 34).

It was therefore concluded that the reaction is efficient and selective for mono-N-alkylation using 1 equiv of $\mathbf{1 a}, 3$ equiv of 2a, 1 equiv of $\mathrm{K}_{2} \mathrm{CO}_{3}, 3$ equiv of $\mathrm{Na}_{2} \mathrm{CO}_{3}$ and $2.5 \mathrm{~mL}$ of methanol at room temperature.

With the optimized conditions established, mono-N-alkylation of a range of aromatic amines with $\mathbf{2 a}$ was performed (Table 2). It was observed that anilines containing electron-donating groups such as Me, and OMe underwent conversion smoothly with excellent selectivity for mono-Nalkylated products with good yields. Electron-withdrawing groups such as $\mathrm{NO}_{2}$ and $\mathrm{COOH}$ at ortho- and para-positions did not show any conversion, presumably due to decreased nucleophilicity of the amino group. However, a nitro group at the meta-position gave high selectivity and moderate yield of 3g. For halogen-substituted anilines, better conversions were seen at all ortho, meta and para positions to give 3h-o with an increase in the selectivity for mono-N-alkylated products. 
Table 1 Selected Reaction Optimisation Observations $s^{\mathrm{a}}$

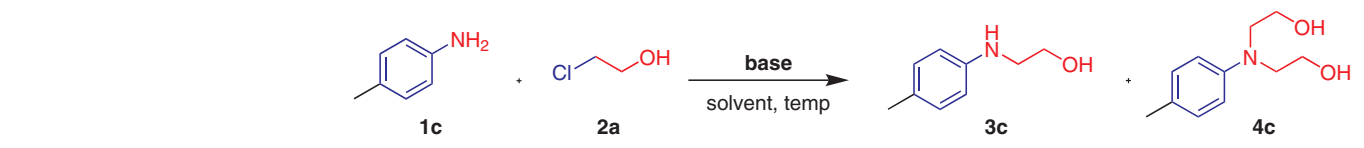

\begin{tabular}{llllll}
\hline Entry & Base (equiv) $\quad$ Additive (equiv) & Solvent (volume, $\mathrm{mL})$ & Time $(\mathrm{h}) \quad \begin{array}{l}\text { Conv. } \\
(\%)^{\mathrm{b}}\end{array}$ & Yield $($ selectivity) $(\%)$ & Ratio of 3/4
\end{tabular}

\begin{tabular}{|c|c|c|c|c|c|c|c|c|}
\hline 1 & triethylamine (1) & - & methanol (2.5) & 12 & 72 & $34(47)$ & $38(53)$ & $47: 53$ \\
\hline 2 & DIPEA (1) & - & methanol (2.5) & 12 & 62 & $27(44)$ & $35(56)$ & $44: 56$ \\
\hline 3 & $\mathrm{Na}_{2} \mathrm{CO}_{3}(1)$ & - & methanol (2.5) & 12 & 1 & $1(100)$ & ND & 100:0 \\
\hline 4 & $\mathrm{NaHCO}_{3}(1)$ & - & methanol (2.5) & 12 & 1 & $1(100)$ & ND & 100:0 \\
\hline 5 & $\mathrm{KHCO}_{3}(1)$ & - & methanol (2.5) & 12 & 1 & $1(100)$ & ND & 100:0 \\
\hline 6 & $\mathrm{~K}_{2} \mathrm{CO}_{3}(1)$ & - & methanol (2.5) & 12 & 78 & $45(58)$ & $33(42)$ & $58: 42$ \\
\hline 7 & $\mathrm{Cs}_{2} \mathrm{CO}_{3}(1)$ & - & methanol (2.5) & 12 & 72 & $20(28)$ & $52(72)$ & $28: 72$ \\
\hline 8 & $\mathrm{~K}_{3} \mathrm{PO}_{4}(1)$ & - & methanol (2.5) & 12 & 75 & $25(33)$ & $50(67)$ & $33: 67$ \\
\hline 9 & $\mathrm{NaOH}(1)$ & - & methanol (2.5) & 12 & 85 & $27(32)$ & $58(68)$ & $32: 68$ \\
\hline 10 & $\mathrm{KOH}(1)$ & - & methanol (2.5) & 12 & 87 & $25(29)$ & $62(71)$ & 29:71 \\
\hline 11 & NaOMe (1) & - & methanol (2.5) & 12 & 95 & $30(32)$ & $65(68)$ & $32: 68$ \\
\hline 12 & $\mathrm{~K}_{2} \mathrm{CO}_{3}(1)$ & - & - & 12 & 75 & $32(43)$ & $43(57)$ & $43: 57$ \\
\hline 13 & $\mathrm{~K}_{2} \mathrm{CO}_{3}(1)$ & $\mathrm{Na}_{2} \mathrm{CO}_{3}(1)$ & methanol (2.5) & 12 & 80 & $49(61)$ & $31(39)$ & $61: 39$ \\
\hline 14 & $\mathrm{~K}_{2} \mathrm{CO}_{3}(1)$ & $\mathrm{Na}_{2} \mathrm{CO}_{3}(2)$ & methanol (2.5) & 12 & 85 & $64(74)$ & $20(26)$ & $74: 26$ \\
\hline 15 & $\mathrm{~K}_{2} \mathrm{CO}_{3}(1)$ & $\mathrm{Na}_{2} \mathrm{CO}_{3}(3)$ & methanol (2.5) & 6 & 91 & $76(84)$ & $15(16)$ & $84: 16$ \\
\hline 16 & $\mathrm{~K}_{2} \mathrm{CO}_{3}(1)$ & $\mathrm{NaHCO}_{3}(3)$ & methanol (2.5) & 12 & 80 & $65(81)$ & $15(19)$ & $81: 19$ \\
\hline 17 & $\mathrm{~K}_{2} \mathrm{CO}_{3}(1)$ & $\mathrm{KHCO}_{3}(3)$ & methanol (2.5) & 12 & 83 & $68(82)$ & $15(18)$ & $82: 18$ \\
\hline 18 & triethylamine (1) & $\mathrm{Na}_{2} \mathrm{CO}_{3}(3)$ & methanol (2.5) & 12 & 75 & $33(44)$ & $42(56)$ & $44: 56$ \\
\hline 19 & $\mathrm{Cs}_{2} \mathrm{CO}_{3}(1)$ & $\mathrm{Na}_{2} \mathrm{CO}_{3}(3)$ & methanol (2.5) & 12 & 88 & $55(63)$ & $33(38)$ & $63: 38$ \\
\hline 20 & $\mathrm{~K}_{3} \mathrm{PO}_{4}(1)$ & $\mathrm{Na}_{2} \mathrm{CO}_{3}(3)$ & methanol (2.5) & 12 & 90 & $36(40)$ & $54(60)$ & $40: 60$ \\
\hline 21 & $\mathrm{~K}_{2} \mathrm{CO}_{3}(1)$ & $\mathrm{Na}_{2} \mathrm{CO}_{3}(3)$ & acetonitrile (2.5) & 24 & 3 & $3(100)$ & ND & $100: 0$ \\
\hline 22 & $\mathrm{~K}_{2} \mathrm{CO}_{3}(1)$ & $\mathrm{Na}_{2} \mathrm{CO}_{3}(3)$ & acetone (2.5) & 24 & 3 & $3(100)$ & ND & $100: 0$ \\
\hline 23 & $\mathrm{~K}_{2} \mathrm{CO}_{3}(1)$ & $\mathrm{Na}_{2} \mathrm{CO}_{3}(3)$ & dichloromethane (2.5) & 24 & 1 & $1(100)$ & ND & $100: 0$ \\
\hline 24 & $\mathrm{~K}_{2} \mathrm{CO}_{3}(1)$ & $\mathrm{Na}_{2} \mathrm{CO}_{3}(3)$ & $\operatorname{THF}(2.5)$ & 24 & 2 & $2(100)$ & ND & $100: 0$ \\
\hline 25 & $\mathrm{~K}_{2} \mathrm{CO}_{3}(1)$ & $\mathrm{Na}_{2} \mathrm{CO}_{3}(3)$ & toluene (2.5) & 24 & 3 & $3(100)$ & ND & $100: 0$ \\
\hline 26 & $\mathrm{~K}_{2} \mathrm{CO}_{3}(1)$ & $\mathrm{Na}_{2} \mathrm{CO}_{3}(3)$ & 1,4-dioxane (2.5) & 24 & ND & ND & ND & $100: 0$ \\
\hline 27 & $\mathrm{~K}_{2} \mathrm{CO}_{3}(1)$ & $\mathrm{Na}_{2} \mathrm{CO}_{3}(3)$ & $\operatorname{DMF}(2.5)$ & 24 & 10 & $10(100)$ & ND & $100: 0$ \\
\hline 28 & $\mathrm{~K}_{2} \mathrm{CO}_{3}(1)$ & $\mathrm{Na}_{2} \mathrm{CO}_{3}(3)$ & DMSO (2.5) & 24 & 7 & $7(100)$ & ND & 100:0 \\
\hline 29 & $\mathrm{~K}_{2} \mathrm{CO}_{3}(1)$ & $\mathrm{Na}_{2} \mathrm{CO}_{3}(3)$ & $\operatorname{NMP}(2.5)$ & 24 & 5 & $5(100)$ & ND & $100: 0$ \\
\hline 30 & $\mathrm{~K}_{2} \mathrm{CO}_{3}(1)$ & $\mathrm{Na}_{2} \mathrm{CO}_{3}(3)$ & ethanol (2.5) & 12 & 89 & $69(78)$ & $20(22)$ & $78: 22$ \\
\hline 31 & $\mathrm{~K}_{2} \mathrm{CO}_{3}(1)$ & $\mathrm{Na}_{2} \mathrm{CO}_{3}(3)$ & isopropyl alcohol (2.5) & 12 & 87 & $62(71)$ & $25(29)$ & $71: 29$ \\
\hline $32^{d}$ & $\mathrm{~K}_{2} \mathrm{CO}_{3}(1)$ & $\mathrm{Na}_{2} \mathrm{CO}_{3}(3)$ & methanol (2.5) & 12 & 55 & $50(91)$ & $5(9)$ & $91: 9$ \\
\hline $33^{e}$ & $\mathrm{~K}_{2} \mathrm{CO}_{3}(1)$ & $\mathrm{Na}_{2} \mathrm{CO}_{3}(3)$ & methanol (2.5) & 12 & 76 & $67(88)$ & $9(12)$ & $88: 12$ \\
\hline $34^{f}$ & $\mathrm{~K}_{2} \mathrm{CO}_{3}(1)$ & $\mathrm{Na}_{2} \mathrm{CO}_{3}(3)$ & methanol (2.5) & 12 & 99 & $70(71)$ & $29(29)$ & $71: 29$ \\
\hline
\end{tabular}

a Reaction conditions ( $0.5 \mathrm{~g}$ scale): $1 \mathrm{c}$ ( $4.67 \mathrm{mmol}, 1$ equiv), 2 a (3 equiv), base (1-3 equiv), additives (1-3 equiv), solvent ( $2.5 \mathrm{~mL})$ at room temperature. All reagent and substrate addition was carried out at room temperature $\left(25^{\circ} \mathrm{C}\right)$.

b Isolated yields.

c Selectivities are given in parentheses.

d 2-Chloroethanol (2a) (1 equiv).

e 2-Chloroethanol (2a) (2 equiv).

${ }^{f}$ Reaction temperature initially at ambient temperature then increased to $40{ }^{\circ} \mathrm{C}$ and then heated to reflux. 
Table 2 Scope of Selective Mono-N-alkylation of Aromatic Amines with 2-Chloroethanol Promoted by $\mathrm{K}_{2} \mathrm{CO}_{3}-\mathrm{Na}_{2} \mathrm{CO}_{3}{ }^{\mathrm{a}}$

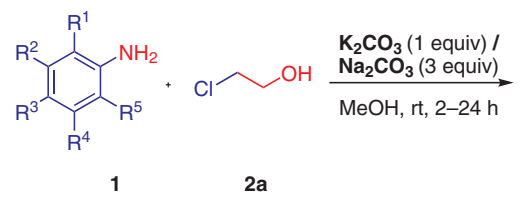<smiles>[R]c1cccc(NCCO)c1[12H]</smiles>

3

mono-N-alkylated

major product selectively<smiles></smiles>

di-N-alkylated

\begin{tabular}{|c|c|c|c|c|c|c|c|c|c|c|}
\hline \multirow[t]{2}{*}{ Entry } & \multirow[t]{2}{*}{$\mathrm{R}^{1}$} & \multirow[t]{2}{*}{$\mathrm{R}^{2}$} & \multirow[t]{2}{*}{$\mathrm{R}^{3}$} & \multirow[t]{2}{*}{$\mathrm{R}^{4}$} & \multirow[t]{2}{*}{$\mathrm{R}^{5}$} & \multirow[t]{2}{*}{ Prod. } & \multirow[t]{2}{*}{ Conv. (\%) } & \multicolumn{2}{|c|}{ Yield $^{\mathrm{b}}$ (selectivity) ${ }^{\mathrm{c}}(\%)$} & \multirow[t]{2}{*}{ Ratio of $3 / 4$} \\
\hline & & & & & & & & 3 & 4 & \\
\hline 1 & $\mathrm{H}$ & $\mathrm{H}$ & $\mathrm{H}$ & $\mathrm{H}$ & $\mathrm{H}$ & $3 a / 4 a$ & 88 & $73(83)$ & $15(17)$ & $83: 17$ \\
\hline 2 & Me & $\mathrm{H}$ & $\mathrm{H}$ & $\mathrm{H}$ & $\mathrm{H}$ & $3 b / 4 b$ & 85 & $75(88)$ & $10(12)$ & $88: 12$ \\
\hline 3 & $\mathrm{H}$ & $\mathrm{H}$ & Me & $\mathrm{H}$ & $\mathrm{H}$ & $3 c / 4 c$ & 91 & $76(84)$ & $15(16)$ & $84: 16$ \\
\hline 4 & Me & $\mathrm{H}$ & Me & $\mathrm{H}$ & $\mathrm{H}$ & $3 d / 4 d$ & 89 & $77(87)$ & $12(13)$ & $87: 13$ \\
\hline 5 & OMe & $\mathrm{H}$ & $\mathrm{H}$ & $\mathrm{H}$ & $\mathrm{H}$ & $3 e / 4 e$ & 85 & $66(81)$ & $15(19)$ & $81: 19$ \\
\hline 6 & $\mathrm{H}$ & $\mathrm{H}$ & OMe & $\mathrm{H}$ & $\mathrm{H}$ & $3 f / 4 f$ & 81 & $80(94)$ & $5(6)$ & $94: 6$ \\
\hline 7 & $\mathrm{H}$ & $\mathrm{NO}_{2}$ & $\mathrm{H}$ & $\mathrm{H}$ & $\mathrm{H}$ & $3 g / 4 g$ & 69 & $64(93)$ & $5(7)$ & $93: 7$ \\
\hline 8 & $\mathrm{Cl}$ & $\mathrm{H}$ & $\mathrm{H}$ & $\mathrm{H}$ & $\mathrm{H}$ & $3 h / 4 h$ & 80 & $72(90)$ & $8(10)$ & $90: 10$ \\
\hline 9 & $\mathrm{H}$ & $\mathrm{Cl}$ & $\mathrm{H}$ & $\mathrm{H}$ & $\mathrm{H}$ & $3 i / 4 i$ & 82 & $69(84)$ & $13(16)$ & $84: 16$ \\
\hline 10 & $\mathrm{H}$ & $\mathrm{H}$ & $\mathrm{Cl}$ & $\mathrm{H}$ & $\mathrm{H}$ & $3 \mathbf{j} / 4 \mathbf{j}$ & 83 & $71(86)$ & $12(14)$ & $86: 14$ \\
\hline 11 & $\mathrm{H}$ & $\mathrm{H}$ & $\mathrm{Br}$ & $\mathrm{H}$ & $\mathrm{H}$ & $3 k / 4 k$ & 80 & $74(93)$ & $6(8)$ & $93: 8$ \\
\hline 12 & $\mathrm{H}$ & $\mathrm{F}$ & $\mathrm{H}$ & $\mathrm{H}$ & $\mathrm{H}$ & $31 / 4 I$ & 91 & $76(84)$ & $15(16)$ & $84: 16$ \\
\hline 13 & $\mathrm{Cl}$ & $\mathrm{Cl}$ & $\mathrm{H}$ & $\mathrm{H}$ & $\mathrm{H}$ & $3 m / 4 m$ & 82 & 79 (96) & $3(4)$ & $96: 4$ \\
\hline 14 & Me & $\mathrm{H}$ & $\mathrm{Br}$ & $\mathrm{H}$ & $\mathrm{H}$ & $3 n / 4 n$ & 84 & $72(86)$ & $12(14)$ & $86: 14$ \\
\hline 15 & Me & $\mathrm{F}$ & $\mathrm{H}$ & $\mathrm{H}$ & $\mathrm{H}$ & $30 / 40$ & 79 & $72(91)$ & 7 (9) & 91:9 \\
\hline
\end{tabular}

a Reaction conditions: Amine 1 ( $0.5 \mathrm{~g}, 1$ equiv), 2 a (3 equiv), $\mathrm{K}_{2} \mathrm{CO}_{3}$ (1 equiv), $\mathrm{Na}_{2} \mathrm{CO}_{3}$ (3 equiv), $\mathrm{MeOH}$ ( $2.5 \mathrm{~mL}, 5$ volume), at room temperature stirring for $2-24 \mathrm{~h}$.

b Isolated yields are given.

c Selectivities are given in parentheses.

The strategy was further extended towards O-alkylation of various substituted phenols $\mathbf{5}$ with $\mathbf{2 a}$ to give moderate to excellent yields (Scheme $1, \mathbf{6 a}-\mathbf{s}$ ). These reactions also demonstrated similar effects on conversion for substrates with substituents having electron-donating or electron- withdrawing groups and steric hindrance at the ortho, meta and para positions. The conditions were also compatible with a range of functional groups, such as alkyl, methoxy, nitro, halide, ester, and aldehyde.

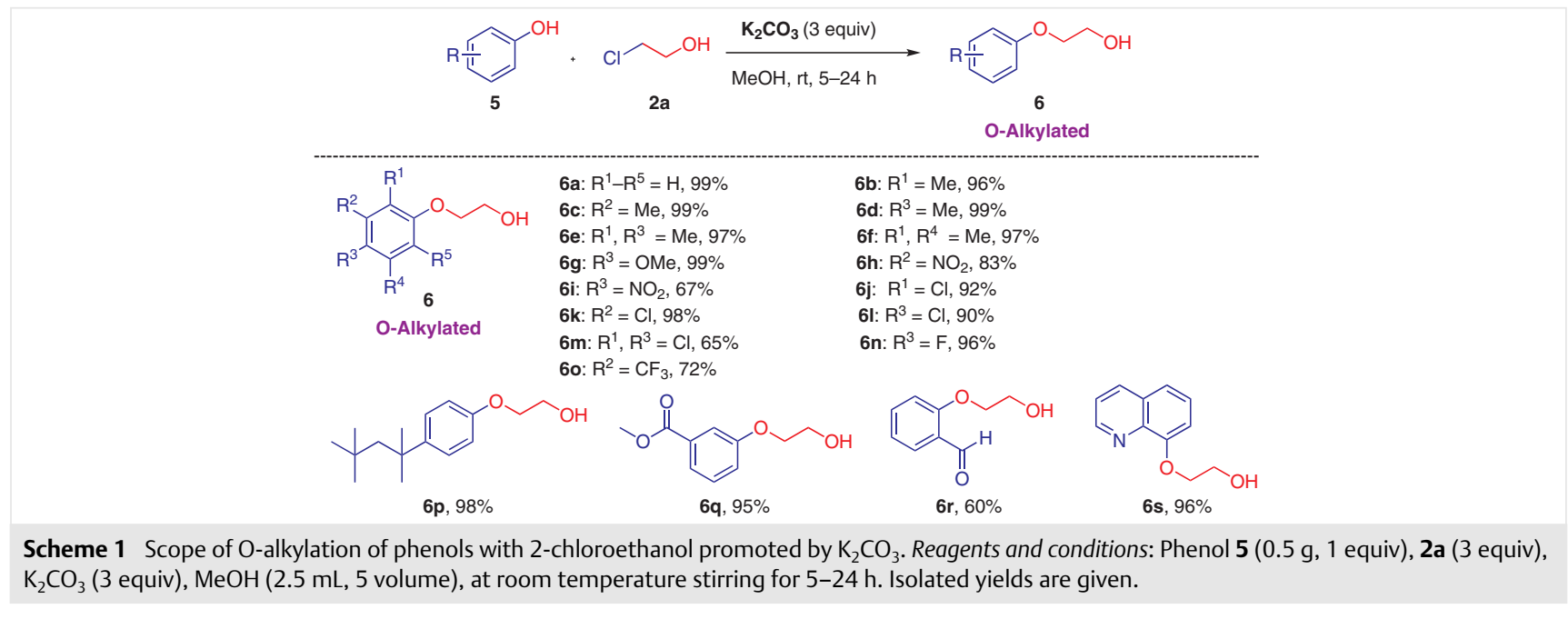


Finally, to demonstrate the synthetic potential of the developed protocol toward the synthesis of commercially important drugs, the formal synthesis of Ticlopidine ${ }^{\circledR,}, 51$ Vildagliptin $^{\circledR},{ }^{52}$ Quetiapine $^{\circledR}, 53$ and Gemfibrozil ${ }^{\circledR 54}$ (Scheme 2) was explored.

The formal synthesis of these four drug molecules was achieved by using the developed protocol with modifications of the reaction conditions as given in Scheme 2. The products formed are in good agreement with those obtained by using the previously reported methods and they were obtained in competitive yields. ${ }^{51-54}$ This protocol will help to improve industrial processes that can be applied in the synthesis of such drugs.

In conclusion, a simple method to attain selective mono-N-alkylation of aromatic and aliphatic primary amines with high selectivity and O-alkylation of phenols with excellent conversion, promoted by $\mathrm{K}_{2} \mathrm{CO}_{3}$ and controlled by $\mathrm{Na}_{2} \mathrm{CO}_{3}$ in methanol at room temperature is presented herein. The mild conditions allow broad functional group tolerance for both amines and phenols. Simple operational and workup procedures make this process applicable for scale-up.
All chemicals were obtained from Sigma-Aldrich, Alfa Aesar, Spectrochem, Avra Synthesis or TCI Europe and used as received without purification. Laboratory grade solvents used for reaction, extraction and column chromatography were purchased from Finar chemicals. The progress of reactions was checked by analytical thin-layer chromatography (TLC Silica gel $60 \mathrm{~F}_{254}$ plates). The plates were visualized first with short-wavelength UV light, followed by staining with iodine.

Melting points were determined with an open capillary tube. GC-MS analyses were recorded with a Shimadzu QP-Ultra 2010 GCMS system with MS detector (EI mode, $70 \mathrm{eV}$ ) and Rxi-624Sil MS column (30 m, $0.32 \mathrm{~mm}$ ID, $1.80 \mu \mathrm{m}$ ). The major signals are quoted in $\mathrm{m} / \mathrm{z}$ with the relative intensity in parentheses. Analyses used an injector temperature of $250{ }^{\circ} \mathrm{C}$; ion source temperature of $200{ }^{\circ} \mathrm{C}$, interface temperature of $260{ }^{\circ} \mathrm{C}$ and column flow $5 \mathrm{~mL} \mathrm{~min}{ }^{-1}$ helium, column initial temperature $\left(T_{0}\right)=60{ }^{\circ} \mathrm{C}$, hold time $(t)=2 \mathrm{~min}$, ramp $=20^{\circ} \mathrm{C} \mathrm{min}^{-1}$, final temperature $\left(T_{1}\right)=240^{\circ} \mathrm{C}$, hold time $(t)=9$ to $39 \mathrm{~min}$. LCMS spectra were recorded with a Shimadzu LCMS-8030 system with a triple quadrupole mass spectrometer in electrospray ionization (ESI) mode.

${ }^{1} \mathrm{H}$ and ${ }^{13} \mathrm{C}$ NMR spectra were recorded in $\mathrm{CDCl}_{3}$ or DMSO- $d_{6}$ with a Bruker Avance-III $500 \mathrm{MHz}$ spectrometer using TMS as an internal standard. The residual solvent signals were used $\left(\mathrm{CDCl}_{3}: \delta_{\mathrm{H}}=7.16-\right.$ $7.32 \mathrm{ppm}$, DMSO- $\left.d_{6}: \delta_{\mathrm{H}}=2.51 \mathrm{ppm}\right)$. Infrared spectra were recorded with a Shimadzu IR MIRacle 10 with diamond ATR.

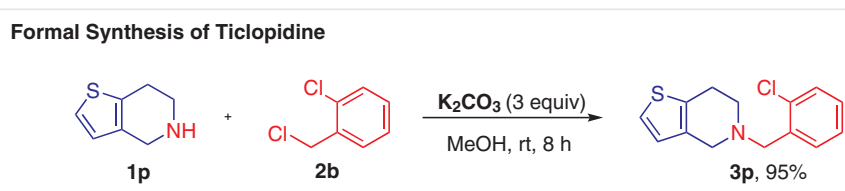

Formal Synthesis of Vildagliptin
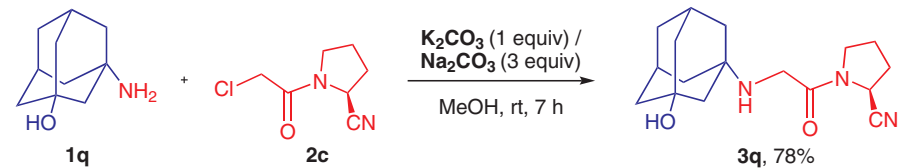

Formal Synthesis of Quetiapine

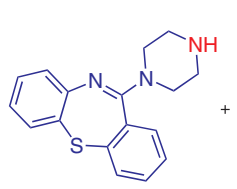

1r

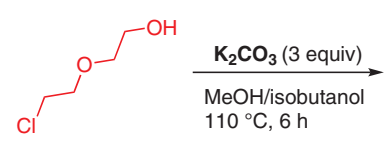

2d<smiles>OCCOCCN1CCN(C2=Nc3ccccc3Sc3ccccc32)CC1</smiles>

Formal Synthesis of Gemfibrozil

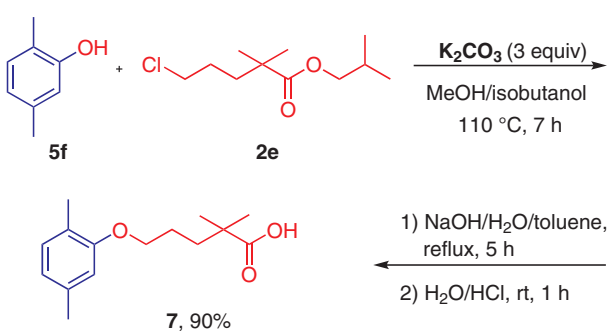<smiles>Cc1ccc(C)c(OCCCC(C)(C)C(=O)OCC(C)C)c1</smiles>

$6 t, 83 \%$

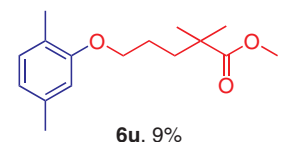

$6 u, 9 \%$

Scheme 2 Formal syntheses of Ticlopidine ${ }^{\circledR}$, Vildagliptin $^{\circledR}$, Quetiapine ${ }^{\circledR}$, and Gemfibrozil ${ }^{\circledR}$ 


\section{Synthesis of 2-(Arylamino)ethanols; Typical Procedure}

To a mixture of amine $\mathbf{1}$ ( $0.5 \mathrm{~g}, 1$ equiv) and 2-chloroethanol 2a (3 equiv) in a round-bottom flask, $\mathrm{K}_{2} \mathrm{CO}_{3}$ ( 1 equiv), $\mathrm{Na}_{2} \mathrm{CO}_{3}$ (3 equiv), and $\mathrm{MeOH}(2.5 \mathrm{~mL})$ were added and the flask was closed with a septum. The mixture was stirred at r.t. and the progress of reaction was monitored by TLC. After completion, the mixture was diluted with cold water $(10 \mathrm{~mL})$ then reaction mass was stirred for 5 minutes and extracted with EtOAc or dichloromethane $(10 \mathrm{~mL})$. The organic layer was then washed with cold water $(10 \mathrm{~mL})$, dried over anhydrous $\mathrm{Na}_{2} \mathrm{SO}_{4}$, filtered and concentrated under vacuum to obtain the crude mixture of products and unreacted amine. Pure mono-N-alkylated amine 3, di-N-alkylated amine $\mathbf{4}$ and unreacted amine $\mathbf{1}$ were obtained after column chromatography.

\section{Synthesis of 2-Aryloxyethanols; Typical Procedure}

To a mixture of phenol $\mathbf{5}$ ( $0.5 \mathrm{~g}, 1$ equiv) and 2-chloroethanol $\mathbf{2 a}$ (3 equiv) in a round-bottom flask, $\mathrm{K}_{2} \mathrm{CO}_{3}$ (3 equiv) and $\mathrm{MeOH}(2.5 \mathrm{~mL}$ ) were added and the flask was closed with a septum. The mixture was stirred at r.t. and the progress of the reaction was monitored by TLC. After completion, the mixture was diluted with cold water $(10 \mathrm{~mL})$ and $1 \mathrm{M}$ aq. $\mathrm{KOH}(10 \mathrm{~mL})$, then the reaction mass was stirred for 5 minutes and extracted with dichloromethane $(10 \mathrm{~mL})$. The organic layer was then washed with $1 \mathrm{M}$ aq. $\mathrm{KOH}(10 \mathrm{~mL})$, dried over anhydrous $\mathrm{Na}_{2} \mathrm{SO}_{4}$ filtered and concentrated under vacuum to obtain the pure product $\mathbf{6}$ without need for further purification.

\section{Formal Synthesis of Ticlopidine ${ }^{\circledR}(3 p)$}

To a mixture of amine $1 \mathbf{p}$ ( $0.5 \mathrm{~g}, 1$ equiv $)$ and alkyl chloride $\mathbf{2 b}(1.2$ equiv) in a round-bottom flask, $\mathrm{K}_{2} \mathrm{CO}_{3}$ (3 equiv) and $\mathrm{MeOH}(5 \mathrm{~mL}$ ) were added and the flask was closed with a septum. The mixture was stirred at r.t. and the progress of reaction was monitored by TLC. After completion, the mixture was diluted with cold water $(10 \mathrm{~mL})$ then stirred for 5 minutes and extracted with EtOAc $(10 \mathrm{~mL})$. The organic layer was washed with cold water $(10 \mathrm{~mL})$, dried over anhydrous $\mathrm{Na}_{2} \mathrm{SO}_{4}$, filtered and concentrated under vacuum to obtain the crude product. Column chromatography afforded pure $\mathbf{3 p}$.

\section{Formal Synthesis of Vildagliptin ${ }^{\circledR}$ (3q)}

To a mixture of amine 1q ( $0.5 \mathrm{~g}$, 1 equiv) and alkyl chloride (2c) (1.2 equiv) in a round-bottom flask, $\mathrm{K}_{2} \mathrm{CO}_{3}$ (1 equiv), $\mathrm{Na}_{2} \mathrm{CO}_{3}$ ( 1 equiv), and $\mathrm{MeOH}(2.5 \mathrm{~mL})$ were added and the flask was closed with a septum. The mixture was stirred at r.t. and the progress of the reaction was monitored by TLC. After completion, the mixture was diluted with $\mathrm{MeOH}(10 \mathrm{~mL})$ and filtered. Evaporation of the solvent gave a residue, which was recrystallized from EtOAc/MeOH (1:1) to obtain pure 3q.

\section{Formal Synthesis of Quetiapine $^{\circledR}$ (3r)}

To a mixture of amine $\mathbf{1 r}$ ( $0.5 \mathrm{~g}, 1$ equiv) and alkyl chloride $\mathbf{2 d}(2.5$ equiv) in a round-bottom flask, $\mathrm{K}_{2} \mathrm{CO}_{3}$ ( 3 equiv), and $\mathrm{MeOH}$ (5 $\mathrm{mL})$ /isobutanol $(5 \mathrm{~mL})$ were added and the flask was fitted with a condenser. The mixture was stirred at $110{ }^{\circ} \mathrm{C}$ and the progress of the reaction was monitored by TLC. After completion, the mixture was diluted with cold water $(10 \mathrm{~mL})$, stirred for 5 minutes and extracted with EtOAc $(10 \mathrm{~mL})$. The organic layer was washed with cold water $(10 \mathrm{~mL})$, dried over anhydrous $\mathrm{Na}_{2} \mathrm{SO}_{4}$, filtered and concentrated under vacuum to obtain crude $3 \mathbf{r}$. Column chromatography afforded pure product.

\section{Formal Synthesis of Gemfibrozil ${ }^{\circledR}$ (7)}

To a mixture of phenol $\mathbf{5 f}$ ( $0.5 \mathrm{~g}, 1$ equiv) and alkyl halide $\mathbf{2 e}$ ( 3 equiv) in a round-bottom flask, $\mathrm{K}_{2} \mathrm{CO}_{3}$ (3 equiv) and $\mathrm{MeOH}(1.5 \mathrm{~mL}$ )/isobutanol $(1.5 \mathrm{~mL})$ were added and the flask was fitted with a condenser. The mixture was stirred at $110{ }^{\circ} \mathrm{C}$ and the progress of reaction was monitored by TLC. After completion, the mixture was diluted with cold water $(10 \mathrm{~mL})$ and $1 \mathrm{M}$ aq. $\mathrm{KOH}(10 \mathrm{~mL})$, stirred for 5 minutes and extracted with dichloromethane $(10 \mathrm{~mL})$. The organic extract was washed with $1 \mathrm{M}$ aq. $\mathrm{KOH}(10 \mathrm{~mL})$, dried over anhydrous $\mathrm{Na}_{2} \mathrm{SO}_{4}$, filtered and concentrated under vacuum to obtain a mixture of products $6 \mathbf{t}$ and $6 \mathbf{u}$.

In a round-bottom flask the mixture of products $6 \mathbf{t}$ and $\mathbf{6 u}$ was dissolved in $10 \mathrm{M}$ aq. $\mathrm{NaOH}$ solution $(10 \mathrm{~mL})$ and toluene $(10 \mathrm{~mL})$ was added. The mixture was heated to reflux for $5 \mathrm{~h}$ and the progress of the reaction was monitored by TLC. After completion, the mixture was cooled, acidified with dilute $\mathrm{HCl}$, stirred for $1 \mathrm{~h}$ and extracted with toluene $(10 \mathrm{~mL})$. The organic extract was dried over anhydrous $\mathrm{Na}_{2} \mathrm{SO}_{4}$, filtered and concentrated under vacuum to obtain pure 7 .

\section{2-(Phenylamino)ethanol (3a)}

Yield: $0.5367 \mathrm{~g}$ (73\%); yellow-brown oil; $R_{f}=0.60$ (hexanes/EtOAc, 65:35).

IR (neat): 3341, 3009, 2940, 2870, 1767, 1597, 1504, 1381, 1319, $1242,1126,1057,872,748,694,625,509 \mathrm{~cm}^{-1}$.

${ }^{1} \mathrm{H}$ NMR $\left(500 \mathrm{MHz}, \mathrm{CDCl}_{3}\right): \delta=7.17\left(\mathrm{dd}, J=8.4,7.4 \mathrm{~Hz}, 2 \mathrm{H}, \mathrm{H}_{\mathrm{Ar}}\right), 6.73$ $\left(\mathrm{t}, J=7.3 \mathrm{~Hz}, 1 \mathrm{H}, \mathrm{H}_{\mathrm{Ar}}\right), 6.62\left(\mathrm{~d}, J=7.7 \mathrm{~Hz}, 2 \mathrm{H}, \mathrm{H}_{\mathrm{Ar}}\right), 3.74(\mathrm{t}, J=5.2 \mathrm{~Hz}$, $2 \mathrm{H}, \mathrm{O}-\mathrm{CH}_{2}$ ), 3.26 (br s, $1 \mathrm{H}, \mathrm{NH}$ ), 3.26 (br s, $\left.1 \mathrm{H}, \mathrm{OH}\right), 3.22(\mathrm{t}, J=5.2 \mathrm{~Hz}$, $2 \mathrm{H}, \mathrm{N}-\mathrm{CH}_{2}$ ).

${ }^{13} \mathrm{C}$ NMR $\left(126 \mathrm{MHz}, \mathrm{CDCl}_{3}\right): \delta=148.05,129.38(2 \mathrm{C}), 118.09,113.44$ (2C), 61.07, 46.22.

GCMS (EI, $70 \mathrm{eV}$ ): $m / z$ calcd for $\mathrm{C}_{8} \mathrm{H}_{11} \mathrm{NO}$ : 137.18; found: 137 .

\section{2-(o-Tolylamino)ethanol (3b)}

Yield: $0.5290 \mathrm{~g}$ (75\%); dark-brown oil; $R_{f}=0.55$ (hexanes/EtOAc, $65: 35)$.

IR (neat): 3379, 2932, 2855, 1759, 1512, 1450, 1381, 1319, 1250, $1134,748,895,525 \mathrm{~cm}^{-1}$.

${ }^{1} \mathrm{H}$ NMR $\left(500 \mathrm{MHz}, \mathrm{CDCl}_{3}\right): \delta=7.16-7.08\left(\mathrm{~m}, 1 \mathrm{H}, \mathrm{H}_{\mathrm{Ar}}\right), 7.06(\mathrm{~d}, J=$ $\left.7.3 \mathrm{~Hz}, 1 \mathrm{H}, \mathrm{H}_{\mathrm{Ar}}\right), 6.68\left(\mathrm{t}, J=7.4 \mathrm{~Hz}, 1 \mathrm{H}, \mathrm{H}_{\mathrm{Ar}}\right), 6.63(\mathrm{~d}, J=8.0 \mathrm{~Hz}, 1 \mathrm{H}$, $\left.\mathrm{H}_{\mathrm{Ar}}\right), 3.82\left(\mathrm{t}, J=5.3 \mathrm{~Hz}, 2 \mathrm{H}, \mathrm{O}-\mathrm{CH}_{2}\right), 3.30\left(\mathrm{t}, J=5.3 \mathrm{~Hz}, 2 \mathrm{H}, \mathrm{O}-\mathrm{CH}_{2}\right)$, 2.88 (br s, $1 \mathrm{H}, \mathrm{NH}$ ), 2.88 (br s, $1 \mathrm{H}, \mathrm{OH}), 2.15\left(\mathrm{~s}, 3 \mathrm{H}, \mathrm{CH}_{3}\right.$ ).

${ }^{13} \mathrm{C}$ NMR $\left(126 \mathrm{MHz}, \mathrm{CDCl}_{3}\right): \delta=146.01,130.29,127.17,122.69$, $117.59,110.18,61.15,46.06,17.53$.

GCMS (EI, $70 \mathrm{eV}$ ): $m / z$ calcd for $\mathrm{C}_{9} \mathrm{H}_{13} \mathrm{NO}$ : 151.21; found: 151 .

\section{2-(p-Tolylamino)ethanol (3c)}

Yield: $0.5365 \mathrm{~g}(76 \%)$; brown solid; $\mathrm{mp} 33-36{ }^{\circ} \mathrm{C} ; R_{f}=0.60$ (hexanes/EtOAc, 65:35).

IR (neat): 3364, 2955, 2909, 2847, 1759, 1612, 1512, 1450, 1381 1296, 1242, 1057, 1018, 980, 941, 918, 810, 718, 694, 617, 540, 463 $\mathrm{cm}^{-1}$.

${ }^{1} \mathrm{H}$ NMR $\left(500 \mathrm{MHz}, \mathrm{CDCl}_{3}\right): \delta=6.91\left(\mathrm{~d}, J=8.2 \mathrm{~Hz}, 2 \mathrm{H}, \mathrm{H}_{\mathrm{Ar}}\right), 6.49(\mathrm{~d}, J=$ $\left.8.4 \mathrm{~Hz}, 2 \mathrm{H}, \mathrm{H}_{\mathrm{Ar}}\right), 3.68\left(\mathrm{t}, J=5.2 \mathrm{~Hz}, 2 \mathrm{H}, \mathrm{O}-\mathrm{CH}_{2}\right), 3.15(\mathrm{t}, J=5.2 \mathrm{~Hz}, 2 \mathrm{H}$, $\mathrm{N}-\mathrm{CH}_{2}$ ), 2.91 (br s, $1 \mathrm{H}, \mathrm{NH}$ ), 2.91 (br s, $\left.1 \mathrm{H}, \mathrm{OH}\right), 2.16\left(\mathrm{~s}, 3 \mathrm{H}, \mathrm{CH}_{3}\right.$ ).

${ }^{13} \mathrm{C}$ NMR $\left(126 \mathrm{MHz}, \mathrm{CDCl}_{3}\right): \delta=145.84,129.84(2 \mathrm{C}), 127.31,113.59$ (2C), 61.22, 46.57, 20.44 .

GCMS (EI, $70 \mathrm{eV}$ ): $m / z$ calcd for $\mathrm{C}_{9} \mathrm{H}_{13} \mathrm{NO}$ : 151.21; found: 151 . 


\section{2-(2,4-Dimethylphenylamino)ethanol (3d)}

Yield: $0.5250 \mathrm{~g}$ (77\%); brown oil; $R_{f}=0.65$ (hexanes/EtOAc, 60:40). IR (neat): 3310, 2909, 2855, 1759, 1612, 1512, 1443, $1381 \mathrm{~cm}^{-1}$. ${ }^{1} \mathrm{H}$ NMR $\left(500 \mathrm{MHz}, \mathrm{CDCl}_{3}\right): \delta=6.85\left(\mathrm{~d}, J=8.1 \mathrm{~Hz}, 1 \mathrm{H}, \mathrm{H}_{\mathrm{Ar}}\right), 6.82(\mathrm{~s}$, $\left.1 \mathrm{H}, \mathrm{H}_{\mathrm{Ar}}\right), 6.48\left(\mathrm{~d}, J=8.1 \mathrm{~Hz}, 1 \mathrm{H}, \mathrm{H}_{\mathrm{Ar}}\right), 3.75\left(\mathrm{t}, J=5.2 \mathrm{~Hz}, 2 \mathrm{H}, \mathrm{O}-\mathrm{CH}_{2}\right)$, $3.22\left(\mathrm{t}, J=5.2 \mathrm{~Hz}, 2 \mathrm{H}, \mathrm{N}-\mathrm{CH}_{2}\right), 2.59(\mathrm{br} \mathrm{s}, 1 \mathrm{H}, \mathrm{NH}), 2.59$ (br s, $1 \mathrm{H}, \mathrm{OH}$ ), $2.15\left(\mathrm{~s}, 3 \mathrm{H}, \mathrm{CH}_{3}\right), 2.06\left(\mathrm{~s}, 3 \mathrm{H}, \mathrm{CH}_{3}\right)$.

${ }^{13} \mathrm{C}$ NMR $\left(126 \mathrm{MHz}, \mathrm{CDCl}_{3}\right): \delta=143.66,131.17,127.36,126.88$, $122.93,110.55,61.20,46.42,20.36,17.47$.

GCMS (EI, $70 \mathrm{eV}$ ): $m / z$ calcd for $\mathrm{C}_{10} \mathrm{H}_{15} \mathrm{NO}$ : 165.23; found: 165 .

\section{2-(2-Methoxyphenylamino)ethanol (3e)}

Yield: $0.4480 \mathrm{~g}$ (66\%); dark-brown oil; $R_{f}=0.40$ (hexanes/EtOAc 50:50).

IR (neat): 3418, 2932, 2878, 1759, 1597, 1512, 1450, 1381, 1242, $1134,1049,903,741,625,586,517 \mathrm{~cm}^{-1}$.

${ }^{1} \mathrm{H}$ NMR $\left(500 \mathrm{MHz}, \mathrm{CDCl}_{3}\right): \delta=6.86\left(\mathrm{td}, J=7.7,1.4 \mathrm{~Hz}, 1 \mathrm{H}, \mathrm{H}_{\mathrm{Ar}}\right), 6.78$ (dd, $\left.J=7.9,1.2 \mathrm{~Hz}, 1 \mathrm{H}, \mathrm{H}_{\mathrm{Ar}}\right), 6.70\left(\mathrm{td}, J=7.8,1.5 \mathrm{~Hz}, 1 \mathrm{H}, \mathrm{H}_{\mathrm{Ar}}\right), 6.65(\mathrm{dd}$, $\left.J=7.8,1.3 \mathrm{~Hz}, 1 \mathrm{H}, \mathrm{H}_{\mathrm{Ar}}\right), 3.83\left(\mathrm{~s}, 3 \mathrm{H}, \mathrm{O}-\mathrm{CH}_{3}\right), 3.822(\mathrm{t}, J=5.3 \mathrm{~Hz}, 2 \mathrm{H}$, $\mathrm{O}-\mathrm{CH}_{2}$ ), 3.30 (t, J = 5.3 Hz, $2 \mathrm{H}, \mathrm{N}-\mathrm{CH}_{2}$ ), 3.08 (br s, $1 \mathrm{H}, \mathrm{NH}$ ), 3.08 (br s, $1 \mathrm{H}, \mathrm{OH}$ )

${ }^{13} \mathrm{C}$ NMR $\left(126 \mathrm{MHz}, \mathrm{CDCl}_{3}\right): \delta=147.20,137.93,121.28,117.17$, $110.38,109.61,61.28,55.44,46.00$.

GCMS (EI, $70 \mathrm{eV}$ ): $m / z$ calcd for $\mathrm{C}_{9} \mathrm{H}_{13} \mathrm{NO}_{2}$ : 167.21; found: 167 .

\section{2-(4-Methoxyphenylamino)ethanol (3f)}

Yield: $0.5436 \mathrm{~g}$ (80\%); dark-brown oil; $R_{f}=0.50$ (hexanes/EtOAc, 40:60).

IR (neat): 3356, 2943, 2832, 2361, 1759, 1508, 1462, 1234, 1180, $1126,1030,899,822,629,567,521 \mathrm{~cm}^{-1}$.

${ }^{1} \mathrm{H}$ NMR $\left(500 \mathrm{MHz}, \mathrm{CDCl}_{3}\right): \delta=6.81-6.75\left(\mathrm{~m}, 2 \mathrm{H}, \mathrm{H}_{\mathrm{Ar}}\right), 6.65-6.57(\mathrm{~m}$, $\left.2 \mathrm{H}, \mathrm{H}_{\mathrm{Ar}}\right), 3.76\left(\mathrm{t}, J=5.2 \mathrm{~Hz}, 2 \mathrm{H}, \mathrm{N}-\mathrm{CH}_{2}\right), 3.74\left(\mathrm{~s}, 3 \mathrm{H}, \mathrm{O}-\mathrm{CH}_{3}\right), 3.20(\mathrm{t}$, $\left.J=5.2 \mathrm{~Hz}, 2 \mathrm{H}, \mathrm{O}-\mathrm{CH}_{2}\right), 3.03(\mathrm{br} \mathrm{s}, 1 \mathrm{H}, \mathrm{OH}), 3.03(\mathrm{br} \mathrm{s}, 1 \mathrm{H}, \mathrm{NH})$.

${ }^{13} \mathrm{C}$ NMR $\left(126 \mathrm{MHz}, \mathrm{CDCl}_{3}\right): \delta=152.53,142.26,114.87$ (4C), 61.16, $55.82,47.22$

GCMS (EI, $70 \mathrm{eV}$ ): $m / z$ calcd for $\mathrm{C}_{9} \mathrm{H}_{13} \mathrm{NO}_{2}$ : 167.21; found: 167 .

\section{2-(3-Nitrophenylamino)ethanol (3g)}

Yield: $0.4221 \mathrm{~g}\left(64 \%\right.$ ); dark-red solid; $\mathrm{mp} 46-50{ }^{\circ} \mathrm{C} ; R_{\mathrm{f}}=0.60$ (hexanes/EtOAc, 50:50).

IR (neat): 3395, 2986, 2955, 2932, 2870, 1805, 1767, 1620, 1582, $1520,1342,1242,1165,1119,1065,988,856,787,733,671,517 \mathrm{~cm}^{-}$ 1 .

${ }^{1} \mathrm{H}$ NMR $\left(500 \mathrm{MHz}, \mathrm{CDCl}_{3}\right): \delta=7.60-7.46\left(\mathrm{~m}, 1 \mathrm{H}, \mathrm{H}_{\mathrm{Ar}}\right), 7.38(\mathrm{~d}, J=$ $\left.4.3 \mathrm{~Hz}, 1 \mathrm{H}, \mathrm{H}_{\mathrm{Ar}}\right), 7.33-7.21\left(\mathrm{~m}, 1 \mathrm{H}, \mathrm{H}_{\mathrm{Ar}}\right), 6.90(\mathrm{dd}, J=8.2,2.2 \mathrm{~Hz}, 1 \mathrm{H}$, $\mathrm{H}_{\mathrm{Ar}}$ ), 4.55 (br s, $1 \mathrm{H}, \mathrm{NH}$ ), 3.87 (t, J = 4.9 Hz, $2 \mathrm{H}, \mathrm{O}-\mathrm{CH}_{2}$ ), 3.33 (br s, $2 \mathrm{H}$, $\left.\mathrm{N}-\mathrm{CH}_{2}\right), 2.45(\mathrm{br} \mathrm{d}, J=54.8 \mathrm{~Hz}, 1 \mathrm{H}, \mathrm{OH})$

${ }^{13} \mathrm{C}$ NMR $\left(126 \mathrm{MHz}, \mathrm{CDCl}_{3}\right): \delta=149.29,149.06,129.78,119.20$, $112.14,106.43,60.86,45.68$

GCMS (EI, $70 \mathrm{eV}$ ): $m / z$ calcd for $\mathrm{C}_{8} \mathrm{H}_{10} \mathrm{~N}_{2} \mathrm{O}_{3}$ : 182.18 ; found: 182 .

\section{2-(2-Chlorophenylamino)ethanol (3h)}

Yield: $0.4847 \mathrm{~g}$ (72\%); yellow oil; $R_{f}=0.50$ (hexanes/EtOAc, 70:30). IR (neat): 3395, 2994, 2940, 2878, 1759, 1597, 1504, 1458, 1373 , $1319,1242,1142,1034,918,895,741,687,532 \mathrm{~cm}^{-1}$.
${ }^{1} \mathrm{H}$ NMR $\left(500 \mathrm{MHz}, \mathrm{CDCl}_{3}\right): \delta=7.28-7.22\left(\mathrm{~m}, 1 \mathrm{H}, \mathrm{H}_{\mathrm{Ar}}\right), 7.12(\mathrm{td}, J=8.2$, $\left.1.5 \mathrm{~Hz}, 1 \mathrm{H}, \mathrm{H}_{\mathrm{Ar}}\right), 6.71-6.59\left(\mathrm{~m}, 2 \mathrm{H}, \mathrm{H}_{\mathrm{Ar}}\right), 4.57$ (br s, $\left.1 \mathrm{H}, \mathrm{NH}\right), 3.81$ (t, $\left.J=5.3 \mathrm{~Hz}, 2 \mathrm{H} \mathrm{O}-\mathrm{CH}_{2}\right), 3.31\left(\mathrm{t}, J=5.3 \mathrm{~Hz}, 2 \mathrm{H} \mathrm{N}-\mathrm{CH}_{2}\right), 2.45$ (br s, $1 \mathrm{H}$, $\mathrm{OH})$.

${ }^{13} \mathrm{C}$ NMR $\left(126 \mathrm{MHz}, \mathrm{CDCl}_{3}\right): \delta=143.96,129.29,127.87,119.60$, $117.72,111.52,61.03,45.75$.

GCMS (EI, $70 \mathrm{eV}$ ): $\mathrm{m} / z$ calcd for $\mathrm{C}_{8} \mathrm{H}_{10} \mathrm{ClNO}$ : 171.62; found: 171 .

\section{2-(3-Chlorophenylamino)ethanol (3i)}

Yield: $0.4643 \mathrm{~g}$ (69\%); dark-brown liquid; $R_{f}=0.50$ (hexanes/EtOAc, 70:30).

IR (neat): 3348, 3341, 2932, 2870, 1767, 1597, 1481, 1404, 1327, $1242,1057,988,934,841,764,687,586 \mathrm{~cm}^{-1}$.

${ }^{1} \mathrm{H}$ NMR $\left(500 \mathrm{MHz}, \mathrm{CDCl}_{3}\right): \delta=6.99\left(\mathrm{t}, J=8.0 \mathrm{~Hz}, 1 \mathrm{H}, \mathrm{H}_{\mathrm{Ar}}\right), 6.61(\mathrm{dd}, J=$ 7.8, $\left.1.4 \mathrm{~Hz}, 1 \mathrm{H}, \mathrm{H}_{\mathrm{Ar}}\right), 6.53\left(\mathrm{t}, J=2.1 \mathrm{~Hz}, 1 \mathrm{H}, \mathrm{H}_{\mathrm{Ar}}\right), 6.42(\mathrm{dd}, J=8.2$, $\left.2.1 \mathrm{~Hz}, 1 \mathrm{H}, \mathrm{H}_{\mathrm{Ar}}\right), 3.72\left(\mathrm{t}, J=5.2 \mathrm{~Hz}, 2 \mathrm{H} \mathrm{O}-\mathrm{CH}_{2}\right), 3.17(\mathrm{t}, J=5.2 \mathrm{~Hz}, 2 \mathrm{H}$, $\mathrm{N}-\mathrm{CH}_{2}$ ), 2.41 (br s, $\left.1 \mathrm{H}, \mathrm{NH}\right), 2.41$ (br s, $1 \mathrm{H}, \mathrm{OH}$ ).

${ }^{13} \mathrm{C}$ NMR $\left(126 \mathrm{MHz}, \mathrm{CDCl}_{3}\right): \delta=149.29,135.06,130.28,117.67$, $112.74,111.59,61.03,45.84$

GCMS (EI, $70 \mathrm{eV}$ ): $m / z$ calcd for $\mathrm{C}_{8} \mathrm{H}_{10} \mathrm{ClNO}$ : 171.62; found: 171

\section{2-(4-Chlorophenylamino)ethanol (3j)}

Yield: $0.4782 \mathrm{~g}\left(71 \%\right.$ ); off-white solid; $\mathrm{mp} 72-75^{\circ} \mathrm{C} ; R_{f}=0.52$ (hexanes/EtOAc, 70:30).

IR (neat): 3186, 2940, 2901, 2855, 1759, 1597, 1497, 1427, 1396, $1312,1265,1242,1119,1057,903 \mathrm{~cm}^{-1}$.

${ }^{1} \mathrm{H}$ NMR $\left(500 \mathrm{MHz}, \mathrm{CDCl}_{3}\right): \delta=7.20-7.06\left(\mathrm{~m}, 2 \mathrm{H}, \mathrm{H}_{\mathrm{Ar}}\right), 6.58-6.52(\mathrm{~m}$, $\left.2 \mathrm{H}, \mathrm{H}_{\mathrm{Ar}}\right), 3.80\left(\mathrm{t}, J=5.2 \mathrm{~Hz}, 2 \mathrm{H}, \mathrm{O}-\mathrm{CH}_{2}\right), 3.23\left(\mathrm{t}, J=5.2 \mathrm{~Hz}, 2 \mathrm{H}, \mathrm{N}-\mathrm{CH}_{2}\right)$, 2.81 (br s, $1 \mathrm{H}, \mathrm{NH}), 2.81$ (br s, $1 \mathrm{H}, \mathrm{OH})$.

${ }^{13} \mathrm{C}$ NMR $\left(126 \mathrm{MHz}, \mathrm{CDCl}_{3}\right): \delta=146.72,129.13(2 \mathrm{C}), 122.43,114.31$ (2C), 61.08, 46.15.

GCMS (EI, $70 \mathrm{eV}$ ): $m / z$ calcd for $\mathrm{C}_{8} \mathrm{H}_{10} \mathrm{ClNO}$; 171.62 ; found: 171

\section{2-(4-Bromophenylamino)ethanol (3k)}

Yield: $0.4648 \mathrm{~g}$ (74\%); off-white solid; $\mathrm{mp} 80-84^{\circ} \mathrm{C} ; R_{f}=0.60$ (hexanes/EtOAc, 60:40).

IR (neat): 3302, 3163, 2932, 2847, 1759, 1589, 1489, 1420, 1389 , $1312,1242,1119,1057,995,903,810,679,594,509 \mathrm{~cm}^{-1}$.

${ }^{1} \mathrm{H}$ NMR $\left(500 \mathrm{MHz}, \mathrm{CDCl}_{3}\right): \delta=7.29-7.07\left(\mathrm{~m}, 2 \mathrm{H}, \mathrm{H}_{\mathrm{Ar}}\right), 6.54-6.37(\mathrm{~m}$, $2 \mathrm{H}, \mathrm{H}_{\mathrm{Ar}}$ ), 3.96 (br s, $\left.1 \mathrm{H}, \mathrm{NH}\right), 3.73\left(\mathrm{t}, J=5.2 \mathrm{~Hz}, 2 \mathrm{H}, \mathrm{O}-\mathrm{CH}_{2}\right), 3.17(\mathrm{t}, J=$ $5.2 \mathrm{~Hz}, 2 \mathrm{H}, \mathrm{N}-\mathrm{CH}_{2}$ ), 1.96 (br s, $1 \mathrm{H}, \mathrm{OH}$ ).

${ }^{13} \mathrm{C}$ NMR (126 MHz, $\left.\mathrm{CDCl}_{3}\right): \delta=147.15,132.00(2 \mathrm{C}), 114.78(2 \mathrm{C})$, 109.45, 61.08, 46.03 .

GCMS (EI, $70 \mathrm{eV}$ ): $m / z$ calcd for $\mathrm{C}_{8} \mathrm{H}_{10}$ BrNO: 216.08; found: 216 .

\section{2-(3-Fluorophenylamino)ethanol (31)}

Yield: $0.5307 \mathrm{~g}$ (76\%); brown oil; $R_{f}=0.55$ (hexanes/EtOAc, 70:30).

IR (neat): 3379, 2932, 2878, 1759, 1620, 1589, 1497, 1450, 1373, 1335, 1242, 1180, 1150, 1111, 1057, 995, 964, 833, 764, 679, 633, $610,579,517 \mathrm{~cm}^{-1}$.

${ }^{1} \mathrm{H} \mathrm{NMR}\left(500 \mathrm{MHz}, \mathrm{CDCl}_{3}\right): \delta=6.99\left(\mathrm{dd}, J=15.0,8.1 \mathrm{~Hz}, 1 \mathrm{H}, \mathrm{H}_{\mathrm{Ar}}\right), 6.30$ $\left(\mathrm{m}, 2 \mathrm{H}, \mathrm{H}_{\mathrm{Ar}}\right), 6.22\left(\mathrm{dt}, J=11.6,2.2 \mathrm{~Hz}, 1 \mathrm{H}, \mathrm{H}_{\mathrm{Ar}}\right), 3.67(\mathrm{t}, J=5.2 \mathrm{~Hz}, 2 \mathrm{H}$, $\mathrm{O}-\mathrm{CH}_{2}$ ), 3.33 (br s, $\left.1 \mathrm{H}, \mathrm{OH}\right), 3.33($ br s, $1 \mathrm{H}, \mathrm{NH}), 3.12(\mathrm{t}, J=5.2 \mathrm{~Hz}, 2 \mathrm{H}$, $\mathrm{N}-\mathrm{CH}_{2}$ ). 
${ }^{13} \mathrm{C}$ NMR $\left(126 \mathrm{MHz}, \mathrm{CDCl}_{3}\right): \delta=163.04(\mathrm{~d}, J=243.3 \mathrm{~Hz}), 148.87(\mathrm{~d}, J=$ $10.6 \mathrm{~Hz}$ ), 129.36 (d, $J=10.3 \mathrm{~Hz}), 108.06$ (d, $J=2.3 \mathrm{~Hz}), 103.15$ (d, $J=$ $22.8 \mathrm{~Hz}), 98.74(\mathrm{~d}, J=25.3 \mathrm{~Hz}), 59.84,44.88$.

GCMS (EI, $70 \mathrm{eV}$ ): $m / z$ calcd for $\mathrm{C}_{8} \mathrm{H}_{10}$ FNO: 155.17; found: 155 .

\section{2-(2,3-Dichlorophenylamino)ethanol (3m)}

Yield: $0.5025 \mathrm{~g}$ (79\%); off-white solid; $\mathrm{mp} 76-80{ }^{\circ} \mathrm{C} ; R_{f}=0.60$ (hexanes/EtOAc, 70:30).

IR (neat): 3387, 3341, 3271, 2955, 2878, 2353, 2322, 1759, 1589, $1497,1450,1412,1319,1250,1111,941,887,748,633,494 \mathrm{~cm}^{-1}$.

${ }^{1} \mathrm{H}$ NMR $\left(500 \mathrm{MHz}, \mathrm{CDCl}_{3}\right): \delta=7.05\left(\mathrm{t}, J=8.1 \mathrm{~Hz}, 1 \mathrm{H}, \mathrm{H}_{\mathrm{Ar}}\right), 6.80(\mathrm{dd}, J=$ 8.0, $1.2 \mathrm{~Hz}, 1 \mathrm{H}, \mathrm{H}_{\mathrm{Ar}}$ ), 6.57 (dd, J = 8.3, $0.9 \mathrm{~Hz}, 1 \mathrm{H}, \mathrm{H}_{\mathrm{Ar}}$ ), 4.78 (br s, $1 \mathrm{H}$, $\mathrm{NH}), 3.86\left(\mathrm{t}, J=5.3 \mathrm{~Hz}, 2 \mathrm{H}, \mathrm{O}-\mathrm{CH}_{2}\right), 3.34\left(\mathrm{t}, J=5.3 \mathrm{~Hz}, 2 \mathrm{H}, \mathrm{N}-\mathrm{CH}_{2}\right)$, $2.01(\mathrm{br} \mathrm{s}, 1 \mathrm{H}, \mathrm{OH})$.

${ }^{13} \mathrm{C}$ NMR $\left(126 \mathrm{MHz}, \mathrm{CDCl}_{3}\right): \delta=145.48,133.00,127.76,118.32$, 117.57, 109.24, 60.97, 45.85.

GCMS (EI, $70 \mathrm{eV}$ ): $m / z$ calcd for $\mathrm{C}_{8} \mathrm{H}_{9} \mathrm{C}_{12} \mathrm{NO}$ : 206.07; found: 206 .

\section{2-(4-Bromo-2-methylphenylamino)ethanol (3n)}

Yield: $0.4456 \mathrm{~g}(72 \%)$; off-white solid; $\mathrm{mp} 74-78^{\circ} \mathrm{C} ; R_{f}=0.52$ (hexanes/EtOAc, 60:40).

IR (neat): 3310, 2916, 2847, 1759, 1597, 1504, 1458, 1396, 1358, $1319,1273,1242,1142,1088,1065,995,856,802 \mathrm{~cm}^{-1}$.

${ }^{1} \mathrm{H}$ NMR $\left(500 \mathrm{MHz}, \mathrm{CDCl}_{3}\right): \delta=7.18\left(\mathrm{dd}, J=8.5,2.2 \mathrm{~Hz}, 1 \mathrm{H}, \mathrm{H}_{\mathrm{Ar}}\right), 7.15$ $\left(\mathrm{d}, J=1.8 \mathrm{~Hz}, 1 \mathrm{H}, \mathrm{H}_{\mathrm{Ar}}\right), 6.46\left(\mathrm{~d}, J=8.5 \mathrm{~Hz}, 1 \mathrm{H}, \mathrm{H}_{\mathrm{Ar}}\right), 3.82(\mathrm{t}, J=5.2 \mathrm{~Hz}$, $2 \mathrm{H}, \mathrm{O}-\mathrm{CH}_{2}$ ), 3.82 (br s, $1 \mathrm{H}, \mathrm{NH}$ ), 3.26 (t, $\mathrm{J}=5.2 \mathrm{~Hz}, 2 \mathrm{H}, \mathrm{N}-\mathrm{CH}_{2}$ ), 2.10 (s, $3 \mathrm{H}, \mathrm{CH}_{3}$ ), 2.10 (br s, $1 \mathrm{H}, \mathrm{OH}$ ).

${ }^{13} \mathrm{C}$ NMR $\left(126 \mathrm{MHz}, \mathrm{CDCl}_{3}\right): \delta=145.12,132.61,129.65,124.79$, 111.57, 109.09, 60.98, 45.97, 17.29.

GCMS (EI, $70 \mathrm{eV}$ ): $m / z$ calcd for $\mathrm{C}_{9} \mathrm{H}_{12}$ BrNO: 230.1; found: 230 .

\section{2-(3-Fluoro-2-methylphenylamino)ethanol (3o)}

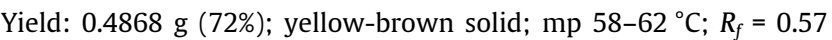
(hexanes/EtOAc, 60:40).

IR (neat): 3395, 3302, 3210, 2932, 2893, 2855, 1759, 1620, 1582, $1520,1450,1381,1319,1288,1234,1134,1049,934,887,864,756$, $694,640,617,579,501 \mathrm{~cm}^{-1}$.

${ }^{1} \mathrm{H}$ NMR $\left(500 \mathrm{MHz}, \mathrm{CDCl}_{3}\right): \delta=6.94\left(\mathrm{dd}, J=15.0,8.0 \mathrm{~Hz}, 1 \mathrm{H}, \mathrm{H}_{\mathrm{Ar}}\right.$ ), 6.38 $\left(\mathrm{t}, J=8.8 \mathrm{~Hz}, 1 \mathrm{H}, \mathrm{H}_{\mathrm{Ar}}\right), 6.31\left(\mathrm{~d}, J=8.2 \mathrm{~Hz}, 1 \mathrm{H}, \mathrm{H}_{\mathrm{Ar}}\right), 3.74(\mathrm{t}, J=5.3 \mathrm{~Hz}$, $2 \mathrm{H}, \mathrm{O}-\mathrm{CH}_{2}$ ), $3.21\left(\mathrm{t}, J=5.2 \mathrm{~Hz}, 2 \mathrm{H}, \mathrm{N}-\mathrm{CH}_{2}\right), 2.71$ (br s, $\left.1 \mathrm{H}, \mathrm{OH}\right), 2.71$ (br s, $1 \mathrm{H}, \mathrm{NH}), 1.96$ (d, $J=1.3 \mathrm{~Hz}, 3 \mathrm{H}$ ).

${ }^{13} \mathrm{C}$ NMR $\left(126 \mathrm{MHz}, \mathrm{CDCl}_{3}\right): \delta=161.50(\mathrm{~d}, J=241.1 \mathrm{~Hz}), 147.72(\mathrm{~d}, J=$ $7.0 \mathrm{~Hz}$ ), 127.20 (d, $J=10.7 \mathrm{~Hz}), 109.16$ (d, $J=18.6 \mathrm{~Hz}), 105.73$ (d, $J=$ $2.4 \mathrm{~Hz}), 104.48$ (d, $J=23.85 \mathrm{~Hz}), 61.04,46.16,8.15$ (d, $J=6.5 \mathrm{~Hz})$.

GCMS (EI, $70 \mathrm{eV}$ ): $m / z$ calcd for $\mathrm{C}_{9} \mathrm{H}_{12}$ FNO: 169.2 ; found: 169 .

\section{2,2'-(Phenylazanediyl)diethanol (4a)}

Yield: $0.1451 \mathrm{~g}$ (15\%); yellow-brown oil; $R_{f}=0.30$ (hexanes/EtOAc, 65:35).

IR (neat): 3287, 2963, 2878, 1767, 1597, 1504, 1358, 1242, 1049, 910 , $856,748,694,602,509 \mathrm{~cm}^{-1}$.

${ }^{1} \mathrm{H}$ NMR (500 MHz, $\left.\mathrm{CDCl}_{3}\right): \delta=7.25-7.16\left(\mathrm{~m}, 2 \mathrm{H}, \mathrm{H}_{\mathrm{Ar}}\right), 6.70(\mathrm{t}, J=$ $\left.7.3 \mathrm{~Hz}, 1 \mathrm{H}, \mathrm{H}_{\mathrm{Ar}}\right), 6.64\left(\mathrm{~d}, J=8.2 \mathrm{~Hz}, 2 \mathrm{H}, \mathrm{H}_{\mathrm{Ar}}\right), 4.41$ (br s, $\left.2 \mathrm{H}, \mathrm{OH}\right), 3.73$ $\left(\mathrm{t}, J=4.9 \mathrm{~Hz}, 4 \mathrm{H}, \mathrm{O}-\mathrm{CH}_{2}\right), 3.48\left(\mathrm{t}, J=4.9 \mathrm{~Hz}, 4 \mathrm{H}, \mathrm{N}-\mathrm{CH}_{2}\right)$.

${ }^{13} \mathrm{C}$ NMR $\left(126 \mathrm{MHz}, \mathrm{CDCl}_{3}\right): \delta=147.80,129.34(2 \mathrm{C}), 116.80,112.48$ (2C), $60.64(2 \mathrm{C}), 55.34(2 \mathrm{C})$.
GCMS (EI, $70 \mathrm{eV}$ ): $m / z$ calcd for $\mathrm{C}_{10} \mathrm{H}_{15} \mathrm{NO}_{2}$ : 181.23; found: 181 .

\section{2,2'-(2-Methylphenylazanediyl)diethanol (4b)}

Yield: $0.0915 \mathrm{~g}$ (10\%); yellow oil; $R_{f}=0.29$ (hexanes/EtOAc, 65:35). IR (neat): 3325, 2940, 2878, 2824, 1759, 1597, 1489, 1443, 1373, $1242,1157,1049,918,872,764,725,594 \mathrm{~cm}^{-1}$.

${ }^{1} \mathrm{H}$ NMR $\left(500 \mathrm{MHz}, \mathrm{CDCl}_{3}\right): \delta=7.18\left(\mathrm{dd}, J=18.2,7.7 \mathrm{~Hz}, 3 \mathrm{H}, \mathrm{H}_{\mathrm{Ar}}\right), 7.05$ (dd, $\left.J=10.2,4.4 \mathrm{~Hz}, 1 \mathrm{H}, \mathrm{H}_{\mathrm{Ar}}\right), 3.58\left(\mathrm{t}, J=5.4 \mathrm{~Hz}, 4 \mathrm{H}, \mathrm{O}-\mathrm{CH}_{2}\right), 3.16(\mathrm{t}, J=$ $5.4 \mathrm{~Hz}, 4 \mathrm{H}, \mathrm{N}-\mathrm{CH}_{2}$ ), 3.01 (br s, $\left.2 \mathrm{H}, \mathrm{OH}\right), 2.35$ (s, $3 \mathrm{H}$ ).

${ }^{13} \mathrm{C}$ NMR $\left(126 \mathrm{MHz}, \mathrm{CDCl}_{3}\right): \delta=149.31,135.71,131.38,126.79$, $125.02,124.17,60.01$ (2C), 56.68 (2C), 18.31.

GCMS (EI, $70 \mathrm{eV}$ ): $m / z$ calcd for $\mathrm{C}_{11} \mathrm{H}_{17} \mathrm{NO}_{2}$ : 195.26; found: 195 .

2,2'-(4-Methylphenylazanediyl)diethanol (4c)

Yield: 0.1365 g (15\%); dark-brown oil; $R_{f}=0.30$ (hexanes/EtOAc, 60:40).

IR (neat): 3296, 2916, 2862, 1759, 1612, 1512, 1443, 1350, 1242, $1180,1042,910,856,802,710,610,571,509 \mathrm{~cm}^{-1}$.

${ }^{1} \mathrm{H}$ NMR $\left(500 \mathrm{MHz}, \mathrm{CDCl}_{3}\right): \delta=6.94\left(\mathrm{~d}, J=8.4 \mathrm{~Hz}, 2 \mathrm{H}, \mathrm{H}_{\mathrm{Ar}}\right), 6.51(\mathrm{~d}, J=$ $8.6 \mathrm{~Hz}, 2 \mathrm{H}, \mathrm{H}_{\mathrm{Ar}}$ ), 3.88 (br s, $\left.2 \mathrm{H}, \mathrm{OH}\right), 3.66\left(\mathrm{t}, J=4.9 \mathrm{~Hz}, 4 \mathrm{H}, \mathrm{O}-\mathrm{CH}_{2}\right)$, $3.38\left(\mathrm{t}, J=4.9 \mathrm{~Hz}, 4 \mathrm{H}, \mathrm{N}-\mathrm{CH}_{2}\right), 2.16\left(\mathrm{~s}, 3 \mathrm{H}, \mathrm{CH}_{3}\right)$.

${ }^{13} \mathrm{C}$ NMR (126 MHz, $\left.\mathrm{CDCl}_{3}\right): \delta=145.75,129.85$ (2C), 126.29, 113.01 (2C), $60.70(2 \mathrm{C}), 55.53(2 \mathrm{C}), 20.20$.

GCMS (EI, $70 \mathrm{eV}$ ): $m / z$ calcd for $\mathrm{C}_{11} \mathrm{H}_{17} \mathrm{NO}_{2}$ : 195.26; found: 195 .

\section{2,2'-(2,4-Dimethylphenylazanediyl)diethanol (4d)}

Yield: $0.1030 \mathrm{~g}$ (12\%); yellow oil; $R_{f}=0.32$ (hexanes/EtOAc, 60:40).

IR (neat): 3356, 2940, 2878, 1497, 1443, 1366, 1265, 1196, 1150, $1042,1150,1042,872,818,571,525 \mathrm{~cm}^{-1}$.

${ }^{1} \mathrm{H}$ NMR $\left(500 \mathrm{MHz}, \mathrm{CDCl}_{3}\right): \delta=7.02\left(\mathrm{~d}, J=8.0 \mathrm{~Hz}, 1 \mathrm{H}, \mathrm{H}_{\mathrm{Ar}}\right), 6.94(\mathrm{~s}, 1 \mathrm{H}$, $\left.\mathrm{H}_{\mathrm{Ar}}\right), 6.91\left(\mathrm{~d}, J=8.1 \mathrm{~Hz}, 1 \mathrm{H}, \mathrm{H}_{\mathrm{Ar}}\right), 3.50\left(\mathrm{t}, J=5.4 \mathrm{~Hz}, 4 \mathrm{H}, \mathrm{O}-\mathrm{CH}_{2}\right), 3.06$ $\left(\mathrm{t}, J=5.4 \mathrm{~Hz}, 4 \mathrm{H}, \mathrm{N}-\mathrm{CH}_{2}\right), 2.81($ br s, $2 \mathrm{H}, \mathrm{OH}), 2.24(\mathrm{~s}, 3 \mathrm{H}), 2.20(\mathrm{~s}$, $3 \mathrm{H})$.

${ }^{13} \mathrm{C}$ NMR $\left(126 \mathrm{MHz}, \mathrm{CDCl}_{3}\right): \delta=146.61,135.51,134.67,132.02$, 127.48, 124.15, 60.07 (2C), 56.99 (2C), 20.82, 18.17.

GCMS (EI, $70 \mathrm{eV}$ ): $m / z$ calcd for $\mathrm{C}_{12} \mathrm{H}_{19} \mathrm{NO}_{2}$ : 209.28; found: 209.

\section{2,2'-(2-Methoxyphenylazanediyl)diethanol (4e)}

Yield: $0.1275 \mathrm{~g}$ (15\%); dark-brown oil; $R_{f}=0.20$ (hexanes/EtOAc, 50:50).

IR (neat): 3372, 2940, 2878, 2839, 1805, 1744, 1589, 1497, 1458, $1373,1242,1157,1049,910,856,748,594,532,494 \mathrm{~cm}^{-1}$.

${ }^{1} \mathrm{H}$ NMR $\left(500 \mathrm{MHz}, \mathrm{CDCl}_{3}\right): \delta=7.20\left(\mathrm{dd}, J=7.8,1.5 \mathrm{~Hz}, 1 \mathrm{H}, \mathrm{H}_{\mathrm{Ar}}\right), 7.14$ $\left(\mathrm{td}, J=8.1,1.6 \mathrm{~Hz}, 1 \mathrm{H}, \mathrm{H}_{\mathrm{Ar}}\right), 6.96\left(\mathrm{td}, J=7.6,1.2 \mathrm{~Hz}, 1 \mathrm{H}, \mathrm{H}_{\mathrm{Ar}}\right), 6.92(\mathrm{dd}$, $\left.J=8.2,0.9 \mathrm{~Hz}, 1 \mathrm{H}, \mathrm{H}_{\mathrm{Ar}}\right), 3.86\left(\mathrm{~s}, 3 \mathrm{H}, \mathrm{O}-\mathrm{CH}_{3}\right), 3.50(\mathrm{t}, J=5.2 \mathrm{~Hz}, 4 \mathrm{H}, \mathrm{O}-$ $\mathrm{CH}_{2}$ ), 3.25 (br s, $2 \mathrm{H}, \mathrm{OH}$ ), 3.20 (t, $J=5.2 \mathrm{~Hz}, 4 \mathrm{H}, \mathrm{N}-\mathrm{CH}_{2}$ ).

${ }^{13} \mathrm{C}$ NMR $\left(126 \mathrm{MHz}, \mathrm{CDCl}_{3}\right): \delta=155.45,138.15,125.93,125.08$, 121.58, 111.64, 59.80, 57.31 (2C), 55.55 (2C).

GCMS (EI, $70 \mathrm{eV}$ ): $m / z$ calcd for $\mathrm{C}_{11} \mathrm{H}_{17} \mathrm{NO}_{3}: 211.26$; found: 211.

2,2'-(4-Methoxyphenylazanediyl)diethanol (4f)

Yield: $0.0430 \mathrm{~g}(5 \%)$; brown oil; $\mathrm{mp} 44-48{ }^{\circ} \mathrm{C} ; R_{f}=0.22$ (hexanes/EtOAc, 40:60).

IR (neat): 3271, 2940, 2909, 2862, 2839, 1759, 1705, 1512, 1443, $1366,1281,1242 \mathrm{~cm}^{-1}$. 
${ }^{1} \mathrm{H}$ NMR $\left(500 \mathrm{MHz}, \mathrm{CDCl}_{3}\right): \delta=6.84-6.78\left(\mathrm{~m}, 2 \mathrm{H}, \mathrm{H}_{\mathrm{Ar}}\right), 6.73-6.66(\mathrm{~m}$, $\left.2 \mathrm{H}, \mathrm{H}_{\mathrm{Ar}}\right), 3.82$ (br s, $\left.2 \mathrm{H}, \mathrm{OH}\right), 3.74\left(\mathrm{~s}, 3 \mathrm{H}, \mathrm{O}-\mathrm{CH}_{3}\right), 3.71(\mathrm{t}, J=5.0 \mathrm{~Hz}$, $\left.4 \mathrm{H}, \mathrm{O}-\mathrm{CH}_{2}\right), 3.39\left(\mathrm{t}, J=5.0 \mathrm{~Hz}, 4 \mathrm{H}, \mathrm{N}-\mathrm{CH}_{2}\right)$.

${ }^{13} \mathrm{C}$ NMR (126 MHz, $\left.\mathrm{CDCl}_{3}\right): \delta=152.22,142.60,115.57$ (2C), 114.88 (2C), 60.51, 55.98, 55.81.

GCMS (EI, $70 \mathrm{eV}$ ): $m / z$ calcd for $\mathrm{C}_{11} \mathrm{H}_{17} \mathrm{NO}_{3}: 211.26$; found: 211 .

\section{2,2'-(4-Nitrophenylazanediyl)diethanol (4g)}

Yield: $0.0414 \mathrm{~g}(5 \%)$; dark-yellow oil; $\mathrm{mp} 92-94{ }^{\circ} \mathrm{C} ; R_{f}=0.30$ (hexanes/EtOAc, 50:50).

IR (neat): 3210, 2955, 2893, 2862, 1759, 1620, 1520, 1481, 1373 , $1335,1281,1234,1173,1126,1080,1034,995,887,849,779,733$, $664,594,540 \mathrm{~cm}^{-1}$.

${ }^{1} \mathrm{H}$ NMR $\left(500 \mathrm{MHz}\right.$, DMSO- $\left.d_{6}\right): \delta=7.47\left(\mathrm{~s}, 1 \mathrm{H}, \mathrm{H}_{\mathrm{Ar}}\right), 7.42-7.34(\mathrm{~m}, 2 \mathrm{H}$, $\left.\mathrm{H}_{\mathrm{Ar}}\right), 7.19-7.09\left(\mathrm{~m}, 1 \mathrm{H}, \mathrm{H}_{\mathrm{Ar}}\right), 4.85(\mathrm{t}, J=5.2 \mathrm{~Hz}, 2 \mathrm{H}, \mathrm{OH}), 3.60(\mathrm{dd}, J=$ $\left.11.3,5.7 \mathrm{~Hz}, 4 \mathrm{H}, \mathrm{O}-\mathrm{CH}_{2}\right), 3.51\left(\mathrm{t}, J=6.1 \mathrm{~Hz}, 4 \mathrm{H}, \mathrm{N}-\mathrm{CH}_{2}\right)$.

${ }^{13} \mathrm{C}$ NMR (126 MHz, DMSO- $\left.d_{6}\right): \delta=149.49,149.48,130.40,118.06$, 109.61, 105.35, 58.34 (2C), 53.51 (2C).

GCMS (EI, $70 \mathrm{eV}$ ): $\mathrm{m} / z$ calcd for $\mathrm{C}_{10} \mathrm{H}_{14} \mathrm{~N}_{2} \mathrm{O}_{4}$ : 226.23; found: 226 .

\section{2,2'-(2-Chlorophenylazanediyl)diethanol (4h)}

Yield: $0.0681 \mathrm{~g}(8 \%) ;$ yellow oil; $R_{f}=0.25$ (hexanes/EtOAc, 70:30). IR (neat): 3294, 3063, 2932, 2870, 1759, 1636, 1589, 1474, 1373, $1242,1119,1049,910,864,756,671,579,525 \mathrm{~cm}^{-1}$.

${ }^{1} \mathrm{H}$ NMR $\left(500 \mathrm{MHz}, \mathrm{CDCl}_{3}\right): \delta=7.40\left(\mathrm{dd}, J=8.0,1.4 \mathrm{~Hz}, 1 \mathrm{H}, \mathrm{H}_{\mathrm{Ar}}\right), 7.31$ (dd, $J=8.0,1.6 \mathrm{~Hz}, 1 \mathrm{H}, \mathrm{H}_{\mathrm{Ar}}$ ), 7.25 (td, $\left.J=7.6,1.5 \mathrm{~Hz}, 1 \mathrm{H}, \mathrm{H}_{\mathrm{Ar}}\right), 7.10$ (td, $\left.J=8.0,1.7 \mathrm{~Hz}, 1 \mathrm{H}, \mathrm{H}_{\mathrm{Ar}}\right), 3.59\left(\mathrm{t}, J=5.3 \mathrm{~Hz}, 4 \mathrm{H}, \mathrm{O}-\mathrm{CH}_{2}\right), 3.27(\mathrm{t}, J=$ $5.3 \mathrm{~Hz}, 4 \mathrm{H}, \mathrm{N}-\mathrm{CH}_{2}$ ), 2.92 (br s, $2 \mathrm{H}, \mathrm{OH}$ ).

${ }^{13} \mathrm{C}$ NMR $\left(126 \mathrm{MHz}, \mathrm{CDCl}_{3}\right): \delta=146.95,132.55,130.58,127.89$, 126.64, 126.30, 59.93 (2C), 56.72 (2C).

GCMS (EI, $70 \mathrm{eV}$ ): $m / z$ calcd for $\mathrm{C}_{10} \mathrm{H}_{14} \mathrm{ClNO}_{2}$ : 215.68; found: 215 .

\section{2,2'-(3-Chlorophenylazanediyl)diethanol (4i)}

Yield: $0.1094 \mathrm{~g}\left(13 \%\right.$ ); white solid; $\mathrm{mp} 88-93^{\circ} \mathrm{C} ; R_{f}=0.25$ (hexanes/EtOAc, 70:30)

IR (neat): 3233, 3140, 2631, 2600, 2399, 2222, 2106, 1967, 1913, $1721,1589,1489,1211,988,833,764,687 \mathrm{~cm}^{-1}$.

${ }^{1} \mathrm{H}$ NMR $\left(500 \mathrm{MHz}, \mathrm{CDCl}_{3}\right): \delta=7.03\left(\mathrm{t}, J=8.2 \mathrm{~Hz}, 1 \mathrm{H}, \mathrm{H}_{\mathrm{Ar}}\right), 6.60(\mathrm{dd}, J=$ 7.8, $\left.1.3 \mathrm{~Hz}, 1 \mathrm{H}, \mathrm{H}_{\mathrm{Ar}}\right), 6.54\left(\mathrm{t}, J=2.2 \mathrm{~Hz}, 1 \mathrm{H}, \mathrm{H}_{\mathrm{Ar}}\right), 6.44(\mathrm{dd}, J=8.5$, $\left.2.3 \mathrm{~Hz}, 1 \mathrm{H}, \mathrm{H}_{\mathrm{Ar}}\right), 4.19$ (br s, $\left.2 \mathrm{H}, \mathrm{OH}\right), 3.70\left(\mathrm{t}, J=4.8 \mathrm{~Hz}, 4 \mathrm{H}, \mathrm{O}-\mathrm{CH}_{2}\right.$ ), $3.43\left(\mathrm{t}, J=4.9 \mathrm{~Hz}, 4 \mathrm{H}, \mathrm{N}-\mathrm{CH}_{2}\right)$.

${ }^{13} \mathrm{C}$ NMR $\left(126 \mathrm{MHz}, \mathrm{CDCl}_{3}\right): \delta=148.92,135.20,130.22,116.67$, $112.32,110.62,60.55(2 \mathrm{C}), 55.30(2 \mathrm{C})$.

GCMS (EI, $70 \mathrm{eV}$ ): $m / z$ calcd for $\mathrm{C}_{10} \mathrm{H}_{14} \mathrm{ClNO}_{2}$ : 215.68; found: 215 .

\section{2,2'-(4-Chlorophenylazanediyl)diethanol (4j)}

Yield: $0.1017 \mathrm{~g}(12 \%)$; off-white solid; $\mathrm{mp} 95-99{ }^{\circ} \mathrm{C} ; R_{f}=0.26$ (hexanes/EtOAc, 70:30).

IR (neat): 3264, 2916, 2862, 1775, 1589, 1489, 1358, 1173, 1065, 910, $856,802,633,563,494 \mathrm{~cm}^{-1}$.

${ }^{1} \mathrm{H}$ NMR $\left(500 \mathrm{MHz}, \mathrm{CDCl}_{3}\right): \delta=7.19-7.11\left(\mathrm{~m}, 2 \mathrm{H}, \mathrm{H}_{\mathrm{Ar}}\right), 6.62-6.55(\mathrm{~m}$, $\left.2 \mathrm{H}, \mathrm{H}_{\mathrm{Ar}}\right), 4.05(\mathrm{br} \mathrm{s}, 2 \mathrm{H}, \mathrm{OH}), 3.77\left(\mathrm{t}, J=4.9 \mathrm{~Hz}, 2 \mathrm{H}, \mathrm{O}-\mathrm{CH}_{2}\right), 3.51(\mathrm{t}, J=$ $\left.4.9 \mathrm{~Hz}, 2 \mathrm{H}, \mathrm{N}-\mathrm{CH}_{2}\right)$.

${ }^{13} \mathrm{C}$ NMR $\left(126 \mathrm{MHz}, \mathrm{CDCl}_{3}\right): \delta=146.42,129.06(2 \mathrm{C}), 121.74,113.70$ (2C), 60.54 (2C), 55.39 (2C).

GCMS (EI, $70 \mathrm{eV}$ ): $m / z$ calcd for $\mathrm{C}_{10} \mathrm{H}_{14} \mathrm{ClNO}_{2}$ : 215.68; found: 215 .

\section{2,2'-(4-Bromophenylazanediyl)diethanol (4k)}

Yield: $0.0456 \mathrm{~g}(6 \%)$; off-white solid; $\mathrm{mp} 88-93^{\circ} \mathrm{C} ; R_{f}=0.29$ (hexanes/EtOAc, 60:40)

IR (neat): 3271, 2947, 2862, 1759, 1582, 1489, 1358, 1242, 1173, $1103,1057,1011,910,849,802,640,548,548,494 \mathrm{~cm}^{-1}$.

${ }^{1} \mathrm{H}$ NMR $\left(500 \mathrm{MHz}, \mathrm{CDCl}_{3}\right): \delta=7.30-7.26\left(\mathrm{~m}, 2 \mathrm{H}, \mathrm{H}_{\mathrm{Ar}}\right), 6.53(\mathrm{~d}, J=$ $9.1 \mathrm{~Hz}, 2 \mathrm{H}, \mathrm{H}_{\mathrm{Ar}}$ ), 4.00 (br s, $\left.2 \mathrm{H}, \mathrm{OH}\right), 3.77$ (t, J = 4.8 Hz, $2 \mathrm{H}, \mathrm{O}-\mathrm{CH}_{2}$ ), $3.50\left(\mathrm{t}, \mathrm{J}=4.8 \mathrm{~Hz}, 2 \mathrm{H}, \mathrm{N}-\mathrm{CH}_{2}\right)$.

${ }^{13} \mathrm{C}$ NMR (126 MHz, $\left.\mathrm{CDCl}_{3}\right): \delta=146.80,131.95$ (2C), $114.16(2 \mathrm{C})$, $108.78,60.49$ (2C), $55.32(2 \mathrm{C})$.

GCMS (EI, $70 \mathrm{eV}$ ): $m / z$ calcd for $\mathrm{C}_{10} \mathrm{H}_{14} \mathrm{BrNO}_{2}$ : 260.13; found: 260 .

\section{2,2'-(3-Fluorophenylazanediyl)diethanol (4l)}

Yield: $0.1340 \mathrm{~g}$ (15\%); dark-brown oil; $R_{f}=0.25$ (hexanes/EtOAc, 70:30).

IR (neat): 3256, 2955, 2878, 1759, 1612, 1497, 1358, 1242, 1157, $1057,849,756,610,517 \mathrm{~cm}^{-1}$

${ }^{1} \mathrm{H}$ NMR $\left(500 \mathrm{MHz}, \mathrm{CDCl}_{3}\right): \delta=7.12\left(\mathrm{dd}, J=15.5,8.2 \mathrm{~Hz}, 1 \mathrm{H}, \mathrm{H}_{\mathrm{Ar}}\right), 6.39$ $\left(\mathrm{m}, 2 \mathrm{H}, \mathrm{H}_{\mathrm{Ar}}\right), 6.32\left(\mathrm{dt}, J=12.9,2.3 \mathrm{~Hz}, 1 \mathrm{H}, \mathrm{H}_{\mathrm{Ar}}\right), 4.62(\mathrm{br} \mathrm{s}, 2 \mathrm{H}, \mathrm{OH})$, $3.75\left(\mathrm{t}, J=4.9 \mathrm{~Hz}, 4 \mathrm{H}, \mathrm{O}-\mathrm{CH}_{2}\right), 3.48\left(\mathrm{t}, J=4.9 \mathrm{~Hz}, 4 \mathrm{H}, \mathrm{N}-\mathrm{CH}_{2}\right)$.

${ }^{13} \mathrm{C}$ NMR $\left(126 \mathrm{MHz}, \mathrm{CDCl}_{3}\right): \delta=150.62(\mathrm{~d}, J=242.6 \mathrm{~Hz}), 149.55(\mathrm{~d}, J=$ $10.5 \mathrm{~Hz}), 130.32(\mathrm{~d}, J=10.4 \mathrm{~Hz}), 107.94(\mathrm{~d}, J=2.0 \mathrm{~Hz}), 103.12(\mathrm{~d}, J=$ $21.6 \mathrm{~Hz}), 99.34(\mathrm{~d}, J=26.2 \mathrm{~Hz}), 60.41(2 \mathrm{C}), 55.35(2 \mathrm{C})$.

GCMS (EI, $70 \mathrm{eV}$ ): $m / z$ calcd for $\mathrm{C}_{10} \mathrm{H}_{14} \mathrm{FNO}_{2}$ : 199.22; found: 199.

\section{2,2'-(2,3-Dichlorophenylazanediyl)diethanol (4m)}

Yield: $0.0235 \mathrm{~g}$ (3\%); yellow oil; $R_{f}=0.30$ (hexanes/EtOAc, 70:30). IR (neat): 3302, 2932, 2870, 2708, 2029, 1921, 1759, 1574, 1450, $1242,1042,918,718,617,532 \mathrm{~cm}^{-1}$.

${ }^{1} \mathrm{H}$ NMR $\left(500 \mathrm{MHz}, \mathrm{CDCl}_{3}\right): \delta=7.29-7.26\left(\mathrm{dd}, J=8.1,1.7 \mathrm{~Hz}, 1 \mathrm{H}, \mathrm{H}_{\mathrm{Ar}}\right)$, $7.23\left(\mathrm{dd}, J=8.1,1.7 \mathrm{~Hz}, 1 \mathrm{H}, \mathrm{H}_{\mathrm{Ar}}\right), 7.18\left(\mathrm{t}, J=7.9 \mathrm{~Hz}, 1 \mathrm{H}, \mathrm{H}_{\mathrm{Ar}}\right), 3.60(\mathrm{t}$, $\left.J=5.3 \mathrm{~Hz}, 4 \mathrm{H}, \mathrm{O}-\mathrm{CH}_{2}\right), 3.29$ (t, $\left.J=5.3 \mathrm{~Hz}, 4 \mathrm{H}, \mathrm{N}-\mathrm{CH}_{2}\right), 2.79$ (br s, $2 \mathrm{H}$, $\mathrm{OH})$.

${ }^{13} \mathrm{C}$ NMR $\left(126 \mathrm{MHz}, \mathrm{CDCl}_{3}\right): \delta=149.01,134.03,131.11,127.57$, $126.93,124.77,59.98(2 \mathrm{C}), 56.25$ (2C).

GCMS (EI, $70 \mathrm{eV}$ ): $m / z$ calcd for $\mathrm{C}_{10} \mathrm{H}_{13} \mathrm{Cl}_{2} \mathrm{NO}_{2}$ : 250.12; found: 250 .

\section{2,2'-(2-Methyl-4-bromophenylazanediyl)diethanol (4n)}

Yield: $0.0889 \mathrm{~g}$ (12\%); dark-brown oil; $R_{f}=0.27$ (hexanes/EtOAc, 60:40).

IR (neat): 3279, 2940, 2878, 1913, 1759, 1643, 1481, 1242, 1057, 818, $571 \mathrm{~cm}^{-1}$.

${ }^{1} \mathrm{H}$ NMR (500 MHz, $\mathrm{CDCl}_{3}$ ): $\delta=7.33\left(\mathrm{~d}, J=2.1 \mathrm{~Hz}, 1 \mathrm{H}, \mathrm{H}_{\mathrm{Ar}}\right), 7.27$ (dd, $\left.J=8.4,2.1 \mathrm{~Hz}, 1 \mathrm{H}, \mathrm{H}_{\mathrm{Ar}}\right), 7.06\left(\mathrm{~d}, J=8.5 \mathrm{~Hz}, 1 \mathrm{H}, \mathrm{H}_{\mathrm{Ar}}\right), 3.56(\mathrm{t}, J=5.3 \mathrm{~Hz}$, $\left.4 \mathrm{H} \mathrm{O}-\mathrm{CH}_{2}\right), 3.35$ (br s, $\left.2 \mathrm{H}, \mathrm{OH}\right), 3.11\left(\mathrm{t}, J=5.3 \mathrm{~Hz}, 4 \mathrm{H}, \mathrm{N}-\mathrm{CH}_{2}\right), 2.30$ (s, $3 \mathrm{H}, \mathrm{CH}_{3}$ ).

${ }^{13} \mathrm{C}$ NMR $\left(126 \mathrm{MHz}, \mathrm{CDCl}_{3}\right): \delta=148.60,138.19,133.99,129.69$, $125.91,117.85,59.83(2 \mathrm{C}), 56.52(2 \mathrm{C}), 18.12$.

GCMS (EI, $70 \mathrm{eV}$ ): $m / z$ calcd for $\mathrm{C}_{11} \mathrm{H}_{16} \mathrm{BrNO}_{2}$ : 274.15; found: 274 .

2,2'-(2-Methyl-4-fluorophenylazanediyl)diethanol (40)

Yield: $0.0588 \mathrm{~g}(7 \%)$; yellow solid; $\mathrm{mp} 54-60{ }^{\circ} \mathrm{C} ; R_{f}=0.28$ (hexanes/EtOAc, 60:40).

IR (neat): 3372, 3310, 2878, 1775, 1582, 1466, 1242, 1049, 787, 718, $633 \mathrm{~cm}^{-1}$. 
${ }^{1} \mathrm{H}$ NMR $\left(500 \mathrm{MHz}, \mathrm{CDCl}_{3}\right): \delta=7.11\left(\mathrm{dd}, J=14.9,7.9 \mathrm{~Hz}, 1 \mathrm{H}, \mathrm{H}_{\mathrm{Ar}}\right), 6.99$ $\left(\mathrm{d}, J=8.0 \mathrm{~Hz}, 1 \mathrm{H}, \mathrm{H}_{\mathrm{Ar}}\right), 6.82\left(\mathrm{t}, J=8.7 \mathrm{~Hz}, 1 \mathrm{H}, \mathrm{H}_{\mathrm{Ar}}\right), 3.59(\mathrm{t}, J=5.4 \mathrm{~Hz}$, $4 \mathrm{H}, \mathrm{O}-\mathrm{CH}_{2}$ ), 3.17 (br s, $\left.2 \mathrm{H}, \mathrm{OH}\right), 3.17\left(\mathrm{t}, J=5.4 \mathrm{~Hz}, 4 \mathrm{H}, \mathrm{N}-\mathrm{CH}_{2}\right), 2.25$ (d, $J=2.4 \mathrm{~Hz}, 3 \mathrm{H}, \mathrm{CH}_{3}$ ).

${ }^{13} \mathrm{C}$ NMR $\left(126 \mathrm{MHz}, \mathrm{CDCl}_{3}\right.$ ): $\delta=162.10(\mathrm{~d}, J=242.5 \mathrm{~Hz}), 151.15$ (d, $J=$ $6.6 \mathrm{~Hz}), 126.68$ (d, $J=10.2 \mathrm{~Hz}), 122.92$ (d, $J=15.9 \mathrm{~Hz}), 119.38$ (d, $J=$ $2.9 \mathrm{~Hz}$ ), 111.50 (d, $J=23.0 \mathrm{~Hz}), 60.01$ (2C), $56.46(2 \mathrm{C}), 9.90$ (d, $J=$ $5.2 \mathrm{~Hz})$.

GCMS (EI, $70 \mathrm{eV}$ ): $m / z$ calcd for $\mathrm{C}_{11} \mathrm{H}_{16} \mathrm{FNO}_{2}$ : 213.25; found: 213.

\section{2-Phenoxyethanol (6a)}

Yield: $0.7265 \mathrm{~g}$ (99\%); colorless oil; $R_{f}=0.55$ (hexanes/EtOAc, 90:10). IR (neat): 3341, 2924, 1759, 1597, 1489, 1242, 1049, 910, 756, 602, $517 \mathrm{~cm}^{-1}$.

${ }^{1} \mathrm{H}$ NMR $\left(500 \mathrm{MHz}, \mathrm{CDCl}_{3}\right): \delta=7.26-7.14\left(\mathrm{~m}, 2 \mathrm{H}, \mathrm{H}_{\mathrm{Ar}}\right), 6.88(\mathrm{t}, J=$ $\left.7.4 \mathrm{~Hz}, 1 \mathrm{H}, \mathrm{H}_{\mathrm{Ar}}\right), 6.82\left(\mathrm{~d}, J=7.9 \mathrm{~Hz}, 2 \mathrm{H}, \mathrm{H}_{\mathrm{Ar}}\right), 3.97(\mathrm{t}, J=4.6 \mathrm{~Hz}, 2 \mathrm{H}, \mathrm{O}-$ $\left.\mathrm{CH}_{2}\right), 3.85\left(\mathrm{t}, J=4.6 \mathrm{~Hz}, 2 \mathrm{H}, \mathrm{O}-\mathrm{CH}_{2}\right), 2.52$ (br s, $1 \mathrm{H}, \mathrm{OH}$ ).

${ }^{13} \mathrm{C}$ NMR $\left(126 \mathrm{MHz}, \mathrm{CDCl}_{3}\right): \delta=158.62,129.52$ (2C), $121.21,114.59$ (2C), 69.14, 61.41.

GC-MS (EI, $70 \mathrm{eV}$ ): $m / z$ calcd for $\mathrm{C}_{8} \mathrm{H}_{10} \mathrm{O}_{2}$ : 138.16; found: 138 .

\section{2-(o-Tolyloxy)ethanol (6b)}

Yield: $0.6758 \mathrm{~g}$ (96\%); pale-yellow liquid; $R_{f}=0.50$ (hexanes/EtOAc, 90:10).

IR (neat): 3302, 2916, 2866, 2361, 1890, 1755, 1655, 1589, 1493 , $1454,1373,1308,1242,1123,1049,918,822,748,714,606 \mathrm{~cm}^{-1}$.

${ }^{1} \mathrm{H}$ NMR $\left(500 \mathrm{MHz}, \mathrm{CDCl}_{3}\right): \delta=7.14\left(\mathrm{t}, J=7.2 \mathrm{~Hz}, 2 \mathrm{H}, \mathrm{H}_{\mathrm{Ar}}\right), 6.87(\mathrm{td}, J=$ $\left.7.5,0.7 \mathrm{~Hz}, 1 \mathrm{H}, \mathrm{H}_{\mathrm{Ar}}\right), 6.81\left(\mathrm{~d}, J=8.3 \mathrm{~Hz}, 1 \mathrm{H}, \mathrm{H}_{\mathrm{Ar}}\right), 4.05(\mathrm{t}, J=4.6 \mathrm{~Hz}$, $2 \mathrm{H}, \mathrm{O}-\mathrm{CH}_{2}$ ), 3.95 (br t, $J=4.6 \mathrm{~Hz}, 2 \mathrm{H}, \mathrm{O}-\mathrm{CH}_{2}$ ), 2.44 (br t, $J=5.2 \mathrm{~Hz}$, $1 \mathrm{H}, \mathrm{OH}), 2.23\left(\mathrm{~s}, 3 \mathrm{H}, \mathrm{CH}_{3}\right)$.

${ }^{13} \mathrm{C}$ NMR $\left(126 \mathrm{MHz}, \mathrm{CDCl}_{3}\right): \delta=156.73,130.85,126.90,126.85$, $120.89,111.3,69.24,61.62,16.27$.

GC-MS (EI, $70 \mathrm{eV}$ ): $m / z$ calcd for $\mathrm{C}_{9} \mathrm{H}_{12} \mathrm{O}_{2}: 152.19$; found: 152 .

\section{2-(m-Tolyloxy)ethanol (6c)}

Yield: $0.6967 \mathrm{~g}$ (99\%); colorless liquid; $R_{f}=0.52$ (hexanes $/$ EtOAc 90:10).

IR (neat): 3351, 2916, 2870, 2361, 2338, 1674, 1585, 1489, 1450, $1377,1261,1157,1049,949,899,856,772,741,691 \mathrm{~cm}^{-1}$.

${ }^{1} \mathrm{H}$ NMR $\left(500 \mathrm{MHz}, \mathrm{CDCl}_{3}\right): \delta=7.08\left(\mathrm{t}, J=7.8 \mathrm{~Hz}, 1 \mathrm{H}, \mathrm{H}_{\mathrm{Ar}}\right), 6.73-6.68$ $\left(\mathrm{m}, 1 \mathrm{H}, \mathrm{H}_{\mathrm{Ar}}\right), 6.68-6.60\left(\mathrm{~m}, 2 \mathrm{H}, \mathrm{H}_{\mathrm{Ar}}\right), 3.96\left(\mathrm{t}, J=4.6 \mathrm{~Hz}, 2 \mathrm{H}, \mathrm{O}-\mathrm{CH}_{2}\right)$, 3.85 (br d, J = 3.9 Hz, $2 \mathrm{H}, \mathrm{O}-\mathrm{CH}_{2}$ ), 2.46 (br s, $1 \mathrm{H}, \mathrm{OH}$ ), 2.24 (s, $3 \mathrm{H}$, $\mathrm{CH}_{3}$.

${ }^{13} \mathrm{C}$ NMR $\left(126 \mathrm{MHz}, \mathrm{CDCl}_{3}\right): \delta=157.57,138.54,128.22,120.90$, $114.36,110.39,68.04,60.38,20.46$.

GC-MS (EI, $70 \mathrm{eV}$ ): $m / z$ calcd for $\mathrm{C}_{9} \mathrm{H}_{12} \mathrm{O}_{2}$ : 152.19; found: 152 .

\section{2-(p-Tolyloxy)ethanol (6d)}

Yield: $0.6967 \mathrm{~g}$ (99\%); colorless liquid; $R_{f}=0.50$ (hexanes/EtOAc, 90:10).

IR (neat): 3337, 2936, 2870, 2361, 2342, 1759, 1690, 1589, 1481, $1447,1373,1277,1246,1161,1134,1061,1038,918,791,745,691$, $602,540,509,474 \mathrm{~cm}^{-1}$.

${ }^{1} \mathrm{H}$ NMR (500 MHz, $\mathrm{CDCl}_{3}$ ): $\delta=7.07\left(\mathrm{t}, J=5.4 \mathrm{~Hz}, 2 \mathrm{H}, \mathrm{H}_{\mathrm{Ar}}\right.$ ), 6.84-6.77 $\left(\mathrm{m}, 2 \mathrm{H}, \mathrm{H}_{\mathrm{Ar}}\right), 4.03\left(\mathrm{t}, J=4.6 \mathrm{~Hz}, 2 \mathrm{H}, \mathrm{O}-\mathrm{CH}_{2}\right), 3.92(\mathrm{t}, J=4.5 \mathrm{~Hz}, 2 \mathrm{H}, \mathrm{O}-$ $\left.\mathrm{CH}_{2}\right), 2.53$ (br s, $\left.1 \mathrm{H}, \mathrm{OH}\right), 2.28\left(\mathrm{~s}, 3 \mathrm{H}, \mathrm{CH}_{3}\right)$.
${ }^{13} \mathrm{C}$ NMR $\left(126 \mathrm{MHz}, \mathrm{CDCl}_{3}\right): \delta=156.5,130.39,130.00$ (2C), 114.47 (2C), 69.34, 61.48, 20.49 .

GC-MS (EI, $70 \mathrm{eV}$ ): $m / z$ calcd for $\mathrm{C}_{9} \mathrm{H}_{12} \mathrm{O}_{2}: 152.19$; found: 152 .

\section{2-(2,4-Dimethylphenoxy)ethanol (6e)}

Yield: $0.6596 \mathrm{~g}\left(97 \%\right.$ ); white solid; $\mathrm{mp} 52-56{ }^{\circ} \mathrm{C} ; R_{f}=0.55$ (hexanes/EtOAc, 90:10).

IR (neat): 3256, 2940, 2916, 2862, 2361, 2330, 1759, 1609, 1504, $1450,1377,1354,1300,1250,1223,1161,1134,1084,1053,934$ $907,883,799,768,710,579,544 \mathrm{~cm}^{-1}$.

${ }^{1} \mathrm{H}$ NMR $\left(500 \mathrm{MHz}, \mathrm{CDCl}_{3}\right): \delta=6.98-6.90\left(\mathrm{~m}, 2 \mathrm{H}, \mathrm{H}_{\mathrm{Ar}}\right), 6.71(\mathrm{~d}, J=$ $\left.8.1 \mathrm{~Hz}, 1 \mathrm{H}, \mathrm{H}_{\mathrm{Ar}}\right), 4.04\left(\mathrm{t}, J=4.6 \mathrm{~Hz}, 2 \mathrm{H}, \mathrm{O}-\mathrm{CH}_{2}\right), 3.94(\mathrm{br} \mathrm{d}, J=4.0 \mathrm{~Hz}$, $2 \mathrm{H}, \mathrm{O}-\mathrm{CH}_{2}$ ), 2.25 (s, $3 \mathrm{H}, \mathrm{CH}_{3}$ ), 2.23 (br s, $\left.1 \mathrm{H}, \mathrm{OH}\right), 2.20$ (s, $3 \mathrm{H}, \mathrm{CH}_{3}$ ).

${ }^{13} \mathrm{C}$ NMR $\left(126 \mathrm{MHz}, \mathrm{CDCl}_{3}\right): \delta=154.61,131.67,130.15,127.06$, 126.64, 111.55, 69.59, 61.71, 20.47, 16.18 .

GC-MS (EI, $70 \mathrm{eV}$ ): $m / z$ calcd for $\mathrm{C}_{10} \mathrm{H}_{14} \mathrm{O}_{2}$ : 166.22; found: 166 .

\section{2-(2,5-Dimethylphenoxy)ethanol (6f)}

Yield: $0.6598 \mathrm{~g}$ (97\%); yellow liquid; $R_{f}=0.55$ (hexanes/EtOAc, 90:10). IR (neat): 3352, 2920, 2870, 2361, 1755, 1582, 1508, 1454, 1412, $1254,1130,1042,949,899,802,718,667,586 \mathrm{~cm}^{-1}$.

${ }^{1} \mathrm{H}$ NMR $\left(500 \mathrm{MHz}, \mathrm{CDCl}_{3}\right): \delta=7.00\left(\mathrm{~d}, J=7.5 \mathrm{~Hz}, 1 \mathrm{H}, \mathrm{H}_{\mathrm{Ar}}\right), 6.68(\mathrm{~d}, J=$ $\left.7.5 \mathrm{~Hz}, 1 \mathrm{H}, \mathrm{H}_{\mathrm{Ar}}\right), 6.63\left(\mathrm{~s}, 1 \mathrm{H}, \mathrm{H}_{\mathrm{Ar}}\right), 4.03\left(\mathrm{t}, J=4.6 \mathrm{~Hz}, 2 \mathrm{H}, \mathrm{O}-\mathrm{CH}_{2}\right), 3.93$ (br d, $J=4.2 \mathrm{~Hz}, 2 \mathrm{H}, \mathrm{O}-\mathrm{CH}_{2}$ ), 2.56 (br s, $\left.1 \mathrm{H}, \mathrm{OH}\right), 2.30\left(\mathrm{~s}, 3 \mathrm{H}, \mathrm{CH}_{3}\right.$ ), $2.18\left(\mathrm{~s}, 3 \mathrm{H}, \mathrm{CH}_{3}\right)$.

${ }^{13} \mathrm{C}$ NMR $\left(126 \mathrm{MHz}, \mathrm{CDCl}_{3}\right): \delta=156.62,136.69,130.57,123.67$, 121.44, 112.49, 69.37, 61.64, 21.41, 15.84 .

GC-MS (EI, $70 \mathrm{eV}$ ): $\mathrm{m} / z$ calcd for $\mathrm{C}_{10} \mathrm{H}_{14} \mathrm{O}_{2}$ : 166.22; found: 166 .

\section{2-(4-Methoxyphenoxy)ethanol (6g)}

Yield: $0.6707 \mathrm{~g}$ (99\%); off-white solid; $\mathrm{mp} 64-70^{\circ} \mathrm{C} ; R_{f}=0.45$ (hexanes/EtOAc, 85:15).

IR (neat): 3291, 3013, 2928, 2870, 2361, 1751, 1504, 1439, 1377, $1292,1227,1088,1030,930,891,826,725,671,571,532,501 \mathrm{~cm}^{-1}$.

${ }^{1} \mathrm{H}$ NMR $\left(500 \mathrm{MHz}, \mathrm{CDCl}_{3}\right): \delta=6.87-6.80\left(\mathrm{~m}, 4 \mathrm{H}, \mathrm{H}_{\mathrm{Ar}}\right), 4.02(\mathrm{t}, J=$ $4.6 \mathrm{~Hz}, 2 \mathrm{H}, \mathrm{O}-\mathrm{CH}_{2}$ ), 3.92 (br dd, $\left.J=8.8,4.6 \mathrm{~Hz}, 2 \mathrm{H}, \mathrm{O}-\mathrm{CH}_{2}\right), 3.76$ (s, $3 \mathrm{H}, \mathrm{O}-\mathrm{CH}_{3}$ ), 2.48 (br s, $1 \mathrm{H}, \mathrm{OH}$ ).

${ }^{13} \mathrm{C}$ NMR $\left(126 \mathrm{MHz}, \mathrm{CDCl}_{3}\right): \delta=154.09,152.78,115.60$ (2C), 114.71 (2C), 69.96, 61.51, 55.73.

GC-MS (EI, $70 \mathrm{eV}$ ): $m / z$ calcd for $\mathrm{C}_{9} \mathrm{H}_{12} \mathrm{O}_{3}$ : 168.19; found: 168

\section{2-(3-Nitrophenoxy)ethanol (6h)}

Yield: $0.5465 \mathrm{~g}\left(83 \%\right.$ ); white solid; $\mathrm{mp} 87-91{ }^{\circ} \mathrm{C} ; R_{f}=0.30$ (hexanes/EtOAc, 90:10).

IR (neat): 3279, 3078, 2936, 2866, 2361, 1763, 1616, 1524, 1450, $1342,1292,1242,1053,953,895,864,791,733,671,613,540,486$ $\mathrm{cm}^{-1}$.

${ }^{1} \mathrm{H}$ NMR $\left(500 \mathrm{MHz}, \mathrm{CDCl}_{3}\right): \delta=7.83$ (ddd, $J=8.2,2.0,0.7 \mathrm{~Hz}, 1 \mathrm{H}, \mathrm{H}_{\mathrm{Ar}}$ ), $7.74\left(\mathrm{t}, J=2.3 \mathrm{~Hz}, 1 \mathrm{H}, \mathrm{H}_{\mathrm{Ar}}\right), 7.44\left(\mathrm{t}, J=8.2 \mathrm{~Hz}, 1 \mathrm{H}, \mathrm{H}_{\mathrm{Ar}}\right), 7.26$ (ddd, $J=$ 8.3, 2.5, $\left.0.6 \mathrm{~Hz}, 1 \mathrm{H}, \mathrm{H}_{\mathrm{Ar}}\right), 4.17$ (t, $\left.J=4.5 \mathrm{~Hz}, 2 \mathrm{H}, \mathrm{O}-\mathrm{CH}_{2}\right), 4.03$ (br d, $J=$ $3.7 \mathrm{~Hz}, 2 \mathrm{H}, \mathrm{O}-\mathrm{CH}_{2}$ ), 2.38 (br s, $1 \mathrm{H}, \mathrm{OH}$ ).

${ }^{13} \mathrm{C}$ NMR $\left(126 \mathrm{MHz}, \mathrm{CDCl}_{3}\right): \delta=159.20,149.13,130.08,121.62$, 116.10, 108.91, 69.95, 61.10.

GC-MS (EI, $70 \mathrm{eV}$ ): $m / z$ calcd for $\mathrm{C}_{8} \mathrm{H}_{9} \mathrm{NO}_{4}$ : 183.16; found: 183 . 


\section{2-(4-Nitrophenoxy)ethanol (6i)}

Yield: $0.4409 \mathrm{~g}$ (67\%); pale-yellow solid; mp $79-83{ }^{\circ} \mathrm{C} ; R_{f}=0.27$ (hexanes/EtOAc, 90:10).

IR (neat): 3252, 2947, 2361, 1759, 1593, 1501, 1331, 1261, 1173 , $1072,1038,914,837,752,687,656,521 \mathrm{~cm}^{-1}$.

${ }^{1} \mathrm{H}$ NMR $\left(500 \mathrm{MHz}, \mathrm{CDCl}_{3}\right): \delta=8.23-8.17\left(\mathrm{~m}, 2 \mathrm{H}, \mathrm{H}_{\mathrm{Ar}}\right), 7.01-6.97(\mathrm{~m}$, $\left.2 \mathrm{H}, \mathrm{H}_{\mathrm{Ar}}\right), 4.21-4.17\left(\mathrm{~m}, 2 \mathrm{H}, \mathrm{O}-\mathrm{CH}_{2}\right), 4.06-4.01\left(\mathrm{~m}, 2 \mathrm{H}, \mathrm{O}-\mathrm{CH}_{2}\right), 2.31$ (br s, $1 \mathrm{H}$ ).

${ }^{13} \mathrm{C}$ NMR $\left(126 \mathrm{MHz}, \mathrm{CDCl}_{3}\right): \delta=163.73,141.71,125.97$ (2C), 114.54 (2C), 70.01, 61.06.

GC-MS (EI, $70 \mathrm{eV}$ ): $m / z$ calcd for $\mathrm{C}_{8} \mathrm{H}_{9} \mathrm{NO}_{4}$ : 183.16; found: 183 .

\section{2-(2-Chlorophenoxy)ethanol (6j)}

Yield: $0.6178 \mathrm{~g}$ (92\%); brown liquid; $R_{f}=0.50$ (hexanes/EtOAc, 85:15). IR (neat): 3383, 2920, 2870, 2361, 2330, 1612, 1508, 1454, 1377, $1288,1238,1177,1042,914,806,737,702,667,637,559,509 \mathrm{~cm}^{-1}$.

${ }^{1} \mathrm{H}$ NMR $\left(500 \mathrm{MHz}, \mathrm{CDCl}_{3}\right): \delta=7.35\left(\mathrm{dd}, J=7.8,1.6 \mathrm{~Hz}, 1 \mathrm{H}, \mathrm{H}_{\mathrm{Ar}}\right), 7.23-$ $7.15\left(\mathrm{~m}, 1 \mathrm{H}, \mathrm{H}_{\mathrm{Ar}}\right), 6.96-6.85\left(\mathrm{~m}, 2 \mathrm{H}, \mathrm{H}_{\mathrm{Ar}}\right), 4.11(\mathrm{t}, J=4.6 \mathrm{~Hz}, 2 \mathrm{H}, \mathrm{O}-$ $\mathrm{CH}_{2}$ ), $3.97\left(\mathrm{t}, J=4.5 \mathrm{~Hz}, 2 \mathrm{H}, \mathrm{O}-\mathrm{CH}_{2}\right), 2.92$ (br s, $1 \mathrm{H}, \mathrm{OH}$ ).

${ }^{13} \mathrm{C}$ NMR $\left(126 \mathrm{MHz}, \mathrm{CDCl}_{3}\right): \delta=154.17,130.32,127.86,123.02$, 121.92, 113.97, 70.67, 61.18.

GC-MS (EI, $70 \mathrm{eV}$ ): $\mathrm{m} / z$ calcd for $\mathrm{C}_{8} \mathrm{H}_{9} \mathrm{ClO}_{2}$ : 172.61; found: 172 .

\section{2-(3-Chlorophenoxy)ethanol (6k)}

Yield: $0.6579 \mathrm{~g}$ (98\%); colorless liquid; $R_{f}=0.60$ (hexanes/EtOAc, $85: 15)$.

IR (neat): 3345, 3314, 2924, 2870, 2361, 2334, 1759, 1597, 1489, $1369,1285,1242,1169,1088,1049,914,826,741,667,563,509 \mathrm{~cm}^{-}$

${ }^{1} \mathrm{H}$ NMR $\left(500 \mathrm{MHz}, \mathrm{CDCl}_{3}\right): \delta=7.85\left(\mathrm{dd}, J=8.1,1.7 \mathrm{~Hz}, 1 \mathrm{H}, \mathrm{H}_{\mathrm{Ar}}\right), 7.59-$ $7.51\left(\mathrm{~m}, 1 \mathrm{H}, \mathrm{H}_{\mathrm{Ar}}\right), 7.11\left(\mathrm{dd}, J=8.4,0.8 \mathrm{~Hz}, 1 \mathrm{H}, \mathrm{H}_{\mathrm{Ar}}\right), 7.08-7.03(\mathrm{~m}, 1 \mathrm{H}$, $\left.\mathrm{H}_{\mathrm{Ar}}\right), 4.24\left(\mathrm{t}, J=4.5 \mathrm{~Hz}, 2 \mathrm{H}, \mathrm{O}-\mathrm{CH}_{2}\right), 3.98(\mathrm{dt}, J=9.3,4.8 \mathrm{~Hz}, 2 \mathrm{H}, \mathrm{O}-$ $\left.\mathrm{CH}_{2}\right), 3.01(\mathrm{t}, J=6.4 \mathrm{~Hz}, 1 \mathrm{H}, \mathrm{OH})$.

${ }^{13} \mathrm{C}$ NMR $\left(126 \mathrm{MHz}, \mathrm{CDCl}_{3}\right): \delta=152.28,139.82,134.50,125.79$, $120.86,115.14,71.33,60.86$

GC-MS (EI, $70 \mathrm{eV}$ ): $m / z$ calcd for $\mathrm{C}_{8} \mathrm{H}_{9} \mathrm{ClO}_{2}$ : 172.61; found: 172 .

\section{2-(4-Chlorophenoxy)ethanol (61)}

Yield: $0.6044 \mathrm{~g}$ (90\%); dark-brown liquid; $R_{f}=0.47$ (hexanes/EtOAc, $85: 15)$.

IR (neat): 3526, 3360, 2943, 2874, 2361, 2330, 1921, 1759, 1690, $1643,1605,1582,1520,1485,1450,1350,1277,1254,1165,1072$, $1038,914,853,775,745,698,667,602,567,517 \mathrm{~cm}^{-1}$.

${ }^{1} \mathrm{H}$ NMR $\left(500 \mathrm{MHz}, \mathrm{CDCl}_{3}\right): \delta=7.26-7.19\left(\mathrm{~m}, 2 \mathrm{H}, \mathrm{H}_{\mathrm{Ar}}\right), 6.87-6.80(\mathrm{~m}$, $\left.2 \mathrm{H}, \mathrm{H}_{\mathrm{Ar}}\right), 4.04\left(\mathrm{t}, J=4.5 \mathrm{~Hz}, 2 \mathrm{H}, \mathrm{O}-\mathrm{CH}_{2}\right), 3.95(\mathrm{dd}, J=9.4,5.3 \mathrm{~Hz}, 2 \mathrm{H}$, $\left.\mathrm{O}-\mathrm{CH}_{2}\right), 2.30(\mathrm{t}, J=6.0 \mathrm{~Hz}, 1 \mathrm{H}, \mathrm{OH})$.

${ }^{13} \mathrm{C}$ NMR $\left(126 \mathrm{MHz}, \mathrm{CDCl}_{3}\right): \delta=157.24,129.42(2 \mathrm{C}), 126.01,115.84$ (2C), 69.53, 61.34 .

GC-MS (EI, $70 \mathrm{eV}$ ): $\mathrm{m} / z$ calcd for $\mathrm{C}_{8} \mathrm{H}_{9} \mathrm{ClO}_{2}$ : 172.61; found: 172 .

\section{2-(2,4-Dichlorophenoxy)ethanol (6m)}

Yield: $0.4129 \mathrm{~g}$ (65\%); yellow liquid; $R_{f}=0.47$ (hexanes/EtOAc, 85:15). IR (neat): 3364, 2928, 2874, 2361, 1967, 1751, 1585, 1389, 1246, $1061,868,802,733,652,571,559 \mathrm{~cm}^{-1}$.
${ }^{1} \mathrm{H}$ NMR $\left(500 \mathrm{MHz}, \mathrm{CDCl}_{3}\right): \delta=7.35\left(\mathrm{~d}, J=2.5 \mathrm{~Hz}, 1 \mathrm{H}, \mathrm{H}_{\mathrm{Ar}}\right), 7.16(\mathrm{dd}$, $\left.J=8.8,2.6 \mathrm{~Hz}, 1 \mathrm{H}, \mathrm{H}_{\mathrm{Ar}}\right), 6.85\left(\mathrm{~d}, J=8.8 \mathrm{~Hz}, 1 \mathrm{H}, \mathrm{H}_{\mathrm{Ar}}\right), 4.09(\mathrm{t}, J=4.5 \mathrm{~Hz}$, $\left.2 \mathrm{H}, \mathrm{O}-\mathrm{CH}_{2}\right), 3.98\left(\mathrm{dd}, J=9.4,5.1 \mathrm{~Hz}, 2 \mathrm{H}, \mathrm{O}-\mathrm{CH}_{2}\right), 2.81(\mathrm{t}, J=6.1 \mathrm{~Hz}$, $1 \mathrm{H}, \mathrm{OH})$.

${ }^{13} \mathrm{C}$ NMR $\left(126 \mathrm{MHz}, \mathrm{CDCl}_{3}\right): \delta=153.02,130.00,127.71,126.25$, $123.70,114.57,71.00,61.10$

GC-MS (EI, $70 \mathrm{eV}$ ): $m / z$ calcd for $\mathrm{C}_{8} \mathrm{H}_{8} \mathrm{Cl}_{2} \mathrm{O}_{2}: 207.05$; found: 207.

\section{2-(4-Fluorophenoxy)ethanol (6n)}

Yield: $0.6683 \mathrm{~g}$ (96\%); colorless liquid; $R_{f}=0.50$ (hexanes/EtOAc, 90:10).

IR (neat): 3375, 2932, 2874, 2361, 1759, 1504, 1454, 1373, 1296, $1204,1042,914,826,745,648,633,563,513 \mathrm{~cm}^{-1}$.

${ }^{1} \mathrm{H}$ NMR $\left(500 \mathrm{MHz}, \mathrm{CDCl}_{3}\right): \delta=7.04-6.91\left(\mathrm{~m}, 2 \mathrm{H}, \mathrm{H}_{\mathrm{Ar}}\right), 6.89-6.80(\mathrm{~m}$, $\left.2 \mathrm{H}, \mathrm{H}_{\mathrm{Ar}}\right), 4.03\left(\mathrm{t}, \mathrm{J}=4.5 \mathrm{~Hz}, 2 \mathrm{H}, \mathrm{O}-\mathrm{CH}_{2}\right), 3.94\left(\mathrm{br} \mathrm{s}, 2 \mathrm{H}, \mathrm{O}-\mathrm{CH}_{2}\right), 2.54$ (br s, $1 \mathrm{H}, \mathrm{OH}$ )

${ }^{13} \mathrm{C}$ NMR $\left(126 \mathrm{MHz}, \mathrm{CDCl}_{3}\right): \delta=157.45(\mathrm{~d}, J=239.0 \mathrm{~Hz}), 154.75(\mathrm{~d}, J=$ $2.1 \mathrm{~Hz}), 115.89$ (d, $J=23.1 \mathrm{~Hz}$ ) (2C), 115.59 (d, $J=8.0 \mathrm{~Hz})(2 \mathrm{C}), 69.89$, 61.37 .

GC-MS (EI, $70 \mathrm{eV}$ ): $m / z$ calcd for $\mathrm{C}_{8} \mathrm{H}_{9} \mathrm{FO}_{2}$ : 156.15; found: 156

\section{2-(3-(Trifluoromethyl)phenoxy)ethanol (6o)}

Yield: $0.4577 \mathrm{~g}$ (72\%); colorless liquid; $R_{f}=0.50$ (hexanes/EtOAc, $85: 15)$.

IR (neat): 3345, 3306, 2932, 2878, 2361, 1755, 1674, 1593, 1493 , 1450, 1323, 1238, 1169, 1119, 1061, 937, 883, 787, 745, 698, 656, $610,517 \mathrm{~cm}^{-1}$.

${ }^{1} \mathrm{H} \mathrm{NMR}\left(500 \mathrm{MHz}, \mathrm{CDCl}_{3}\right): \delta=7.38\left(\mathrm{t}, J=8.0 \mathrm{~Hz}, 1 \mathrm{H}, \mathrm{H}_{\mathrm{Ar}}\right), 7.22(\mathrm{~d}, J=$ $\left.7.7 \mathrm{~Hz}, 1 \mathrm{H}, \mathrm{H}_{\mathrm{Ar}}\right), 7.14\left(\mathrm{~s}, 1 \mathrm{H}, \mathrm{H}_{\mathrm{Ar}}\right), 7.08$ (dd, $J=8.3,2.3 \mathrm{~Hz}, 1 \mathrm{H}, \mathrm{H}_{\mathrm{Ar}}$ ), $4.10\left(\mathrm{t}, J=4.5 \mathrm{~Hz}, 2 \mathrm{H}, \mathrm{O}-\mathrm{CH}_{2}\right), 3.98$ (br dd, $J=9.2,4.9 \mathrm{~Hz}, 2 \mathrm{H}, \mathrm{O}-\mathrm{CH}_{2}$ ), 2.55 (br t, $J=5.8 \mathrm{~Hz}, 1 \mathrm{H}, \mathrm{OH}$ ).

${ }^{13} \mathrm{C}$ NMR (126 MHz, $\left.\mathrm{CDCl}_{3}\right): \delta=158.76,131.89(\mathrm{q}, J=32.4 \mathrm{~Hz}), 130.08$, $123.92(\mathrm{q}, J=408.2 \mathrm{~Hz}), 118.01(\mathrm{~d}, J=1.0 \mathrm{~Hz}), 117.80(\mathrm{q}, J=3.9 \mathrm{~Hz})$, $111.36(\mathrm{q}, J=3.8 \mathrm{~Hz}), 69.52,61.20$.

GC-MS (EI, $70 \mathrm{eV}$ ): $\mathrm{m} / z$ calcd for $\mathrm{C}_{9} \mathrm{H}_{9} \mathrm{~F}_{3} \mathrm{O}_{2}$ : 206.16; found: 206 .

\section{2-(4-(2,4,4-Trimethylpentan-2-yl)phenoxy)ethanol (6p)}

Yield: $0.5948 \mathrm{~g}$ (98\%); pale-yellow liquid; $R_{f}=0.55$ (hexanes/EtOAc, 90:10).

IR (neat): 3325, 2951, 2870, 2361, 2268, 2118, 2064, 2041, 1944, $1883,1759,1639,1609,1512,1458,1366,1242,1042,918,829,683$, $586,517 \mathrm{~cm}^{-1}$

${ }^{1} \mathrm{H}$ NMR $\left(500 \mathrm{MHz}, \mathrm{CDCl}_{3}\right): \delta=7.28-7.25\left(\mathrm{~m}, 2 \mathrm{H}, \mathrm{H}_{\mathrm{Ar}}\right), 6.87-6.81(\mathrm{~m}$, $\left.2 \mathrm{H}, \mathrm{H}_{\mathrm{Ar}}\right), 4.06\left(\mathrm{t}, \mathrm{J}=4.6 \mathrm{~Hz}, 2 \mathrm{H}, \mathrm{O}-\mathrm{CH}_{2}\right), 3.94\left(\mathrm{br} \mathrm{s}, 2 \mathrm{H}, \mathrm{O}-\mathrm{CH}_{2}\right), 2.33$ (br s, $1 \mathrm{H}, \mathrm{OH}), 1.70\left(\mathrm{~s}, 2 \mathrm{H}, \mathrm{CH}_{2}\right), 1.34\left(\mathrm{~s}, 6 \mathrm{H}, \mathrm{CH}_{3}\right), 0.71\left(\mathrm{~s}, 9 \mathrm{H}, \mathrm{CH}_{3}\right)$.

${ }^{13} \mathrm{C}$ NMR (126 MHz, $\mathrm{CDCl}_{3}$ ): $\delta=156.23,142.74,127.16$ (2C), 113.75 (2C), 69.12, 61.55, 56.99, 37.98, 32.35, 31.80 (3C), 31.71 (2C).

GC-MS (EI, $70 \mathrm{eV}$ ): $m / z$ calcd for $\mathrm{C}_{16} \mathrm{H}_{26} \mathrm{O}_{2}$ : 250.38; found: 250 .

\section{Methyl 3-(2-Hydroxyethoxy)benzoate (6q)}

Yield: $0.6123 \mathrm{~g}$ (95\%); colorless liquid; $R_{f}=0.30$ (hexanes/EtOAc, 90:10).

IR (neat): 3483, 3406, 3352, 3337, 2943, 2866, 2361, 1717, 1585, $1443,1277,1045,926,895,756,683,610,555,517 \mathrm{~cm}^{-1}$. 
${ }^{1} \mathrm{H}$ NMR $\left(500 \mathrm{MHz}, \mathrm{CDCl}_{3}\right): \delta=7.66-7.64\left(\mathrm{~m}, 1 \mathrm{H}, \mathrm{H}_{\mathrm{Ar}}\right), 7.57(\mathrm{dd}, J=$ 2.4, $\left.1.6 \mathrm{~Hz}, 1 \mathrm{H}, \mathrm{H}_{\mathrm{Ar}}\right), 7.34\left(\mathrm{t}, J=8.0 \mathrm{~Hz}, 1 \mathrm{H}, \mathrm{H}_{\mathrm{Ar}}\right), 7.12(\mathrm{dd}, J=7.9$, $3.0 \mathrm{~Hz}, 1 \mathrm{H}, \mathrm{H}_{\mathrm{Ar}}$ ), $4.13\left(\mathrm{t}, J=4.6 \mathrm{~Hz}, 2 \mathrm{H}, \mathrm{O}-\mathrm{CH}_{2}\right.$ ), 3.98 (br dd, $J=9.0$, $4.8 \mathrm{~Hz}, 2 \mathrm{H}, \mathrm{O}-\mathrm{CH}_{2}$ ), 3.91 (s, $\left.3 \mathrm{H}, \mathrm{O}-\mathrm{CH}_{3}\right), 2.48$ (br t, J = 5.7 Hz, $1 \mathrm{H}, \mathrm{OH}$ ). ${ }^{13} \mathrm{C}$ NMR $\left(126 \mathrm{MHz}, \mathrm{CDCl}_{3}\right): \delta=166.94,158.62,131.46,129.51$, $122.39,119.98,114.72,69.44,61.30,52.24$.

GC-MS (EI, $70 \mathrm{eV}$ ): $m / z$ calcd for $\mathrm{C}_{10} \mathrm{H}_{12} \mathrm{O}_{4}$ : 196.2; found: 196.

\section{2-(2-Hydroxyethoxy)benzaldehyde (6r)}

Yield: $0.4080 \mathrm{~g}(60 \%) ;$ yellow oil; $R_{f}=0.30$ (hexanes/EtOAc, 90:10). IR (neat): 3379, 3364, 2932, 2870, 2361, 1759, 1678, 1597, 1454, $1396,1242,1161,1045,918,833,760,656,517 \mathrm{~cm}^{-1}$.

${ }^{1} \mathrm{H}$ NMR (500 MHz, $\mathrm{CDCl}_{3}$ ): $\delta=10.43(\mathrm{~s}, 1 \mathrm{H}, \mathrm{CHO}), 7.80$ (dd, $J=7.7$, $\left.1.8 \mathrm{~Hz}, 1 \mathrm{H}, \mathrm{H}_{\mathrm{Ar}}\right), 7.60-7.50\left(\mathrm{~m}, 1 \mathrm{H}, \mathrm{H}_{\mathrm{Ar}}\right), 7.05\left(\mathrm{t}, J=7.5 \mathrm{~Hz}, 1 \mathrm{H}, \mathrm{H}_{\mathrm{Ar}}\right)$, $7.00\left(\mathrm{~d}, J=8.4 \mathrm{~Hz}, 1 \mathrm{H}, \mathrm{H}_{\mathrm{Ar}}\right), 4.20\left(\mathrm{t}, J=4.6 \mathrm{~Hz}, 2 \mathrm{H}, \mathrm{O}-\mathrm{CH}_{2}\right), 4.03(\mathrm{t}, J=$ $4.5 \mathrm{~Hz}, 2 \mathrm{H}, \mathrm{O}-\mathrm{CH}_{2}$ ), 3.13 (br s, $1 \mathrm{H}, \mathrm{OH}$ ).

${ }^{13} \mathrm{C}$ NMR $\left(126 \mathrm{MHz}, \mathrm{CDCl}_{3}\right): \delta=190.24,160.87,136.08,129.71$, 125.00, 121.13, 112.94, 70.21, 61.07.

GC-MS (EI, $70 \mathrm{eV}$ ): $m / z$ calcd for $\mathrm{C}_{9} \mathrm{H}_{10} \mathrm{O}_{3}$ : 166.17; found: 166 .

\section{2-(Quinolin-8-yloxy)ethanol (6s)}

Yield: $0.6256 \mathrm{~g}$ (96\%); pale-brown solid; $\mathrm{mp} 112-116{ }^{\circ} \mathrm{C} ; R_{f}=0.40$ (hexanes/EtOAc, 40:60).

IR (neat): 3387, 2994, 2855, 1759, 1666, 1574, 1504, 1450, 1373 , $1319,1250,1119,1072,903,764,733,633,571,532 \mathrm{~cm}^{-1}$.

${ }^{1} \mathrm{H}$ NMR $\left(500 \mathrm{MHz}, \mathrm{CDCl}_{3}\right): \delta=8.82\left(\mathrm{dd}, J=4.2,1.6 \mathrm{~Hz}, 1 \mathrm{H}, \mathrm{H}_{\mathrm{Ar}}\right), 8.13$ $\left(\mathrm{dd}, J=8.3,1.5 \mathrm{~Hz}, 1 \mathrm{H}, \mathrm{H}_{\mathrm{Ar}}\right), 7.45\left(\mathrm{t}, J=7.9 \mathrm{~Hz}, 1 \mathrm{H}, \mathrm{H}_{\mathrm{Ar}}\right), 7.41-7.37(\mathrm{~m}$, $\left.2 \mathrm{H}, \mathrm{H}_{\mathrm{Ar}}\right), 7.07\left(\mathrm{~d}, J=7.5 \mathrm{~Hz}, 1 \mathrm{H}, \mathrm{H}_{\mathrm{Ar}}\right), 5.87(\mathrm{br} \mathrm{s}, 1 \mathrm{H}, \mathrm{OH}), 4.26(\mathrm{t}, J=$ $\left.4.4 \mathrm{~Hz}, 2 \mathrm{H}, \mathrm{O}-\mathrm{CH}_{2}\right), 4.11\left(\mathrm{t}, J=4.4 \mathrm{~Hz}, 2 \mathrm{H}, \mathrm{O}-\mathrm{CH}_{2}\right)$.

${ }^{13} \mathrm{C}$ NMR $\left(126 \mathrm{MHz}, \mathrm{CDCl}_{3}\right): \delta=154.37,148.67,139.85,136.54$, $129.49,127.02,121.70,119.89,109.61,70.96,60.83$.

GC-MS (EI, $70 \mathrm{eV}$ ): $m / z$ calcd for $\mathrm{C}_{11} \mathrm{H}_{11} \mathrm{NO}_{2}$ : 189.21; found: 189 .

\section{5-(2-Chlorobenzyl)-4,5,6,7-tetrahydrothieno[3,2-c]pyridine (3p) $)^{55}$} Yield: $0.9013 \mathrm{~g}$ (95\%); colorless oil; $R_{f}=0.70$ (hexanes/EtOAc, 85:15). IR (neat): 3055, 2901, 2770, 2275, 2361, 2060, 1921, 1763, 1674, 1566, 1443, 1358, 1254, 1165, 1107, 1038, 903, 837, 752, 702, 590 $\mathrm{cm}^{-1}$.

${ }^{1} \mathrm{H}$ NMR $\left(500 \mathrm{MHz}, \mathrm{CDCl}_{3}\right): \delta=7.45\left(\mathrm{dd}, J=7.6,1.6 \mathrm{~Hz}, 1 \mathrm{H}, \mathrm{H}_{\mathrm{Ar}}\right), 7.27$ (dd, $\left.J=7.8,1.3 \mathrm{~Hz}, 1 \mathrm{H}, \mathrm{H}_{\mathrm{Ar}}\right), 7.16-7.08\left(\mathrm{~m}, 2 \mathrm{H}, \mathrm{H}_{\mathrm{Ar}}\right), 6.97(\mathrm{~d}, J=5.1 \mathrm{~Hz}$, $\left.1 \mathrm{H}, \mathrm{H}_{\mathrm{Ar}}\right), 6.61\left(\mathrm{~d}, J=5.1 \mathrm{~Hz}, 1 \mathrm{H}, \mathrm{H}_{\mathrm{Ar}}\right), 3.73\left(\mathrm{~s}, 2 \mathrm{H}, \mathrm{N}-\mathrm{CH}_{2}\right), 3.54(\mathrm{~s}, 2 \mathrm{H}$, $\left.\mathrm{N}-\mathrm{CH}_{2}\right), 2.80\left(\mathrm{~d}, J=5.0 \mathrm{~Hz}, 2 \mathrm{H}, \mathrm{N}-\mathrm{CH}_{2}\right), 2.78-2.74\left(\mathrm{~m}, 2 \mathrm{H}, \mathrm{CH}_{2}\right)$.

${ }^{13} \mathrm{C}$ NMR $\left(126 \mathrm{MHz}, \mathrm{CDCl}_{3}\right): \delta=135.06,133.16,132.85,132.35$, $129.55,128.38,127.13,125.66,124.20,121.56,57.40,52.06,49.68$, 24.48 .

GCMS (EI, $70 \mathrm{eV}$ ): $m / z$ calcd for $\mathrm{C}_{14} \mathrm{H}_{14} \mathrm{ClNS}$ : 263.79; found: 263 .

\section{(2S)-1-[2-[(3-Hydroxy-1-adamantyl)amino]acetyl]pyrrolidine-2-} carbonitrile (3q) $)^{56}$

Yield: $0.7075 \mathrm{~g}$ (78\%); off-white solid; $\mathrm{mp} 112-116{ }^{\circ} \mathrm{C} ; R_{f}=0.50$ (EtOAc/MeOH, 90:10).

IR (neat): 3291, 2913, 2847, 2361, 2330, 1755, 1655, 1547, 1512, 1450, 1404, 1354, 1308, 1250, 1188, 1153, 1119, 1034, 964, 910, 826, $791,671,637,602,552,513,463 \mathrm{~cm}^{-1}$.
${ }^{1} \mathrm{H}$ NMR (500 MHz, $\left.\mathrm{CDCl}_{3}\right): \delta=4.89-4.75(\mathrm{~m}, 1 \mathrm{H}, \mathrm{N}-\mathrm{CH}), 3.70-3.58$ $(\mathrm{m}, 1 \mathrm{H}, \mathrm{CH}), 3.52-3.38\left(\mathrm{~m}, 3 \mathrm{H}, \mathrm{CH}, \mathrm{CH}_{2}\right), 2.40-2.12\left(\mathrm{~m}, 8 \mathrm{H}, \mathrm{CH}_{2}\right)$, $1.68-1.49\left(\mathrm{~m}, 12 \mathrm{H}, \mathrm{CH}_{2}\right)$.

${ }^{13} \mathrm{C} \mathrm{NMR}\left(126 \mathrm{MHz}, \mathrm{CDCl}_{3}\right): \delta=170.60,170,47,118.33,118.28,69.45$, 53.76, 53.46, 49.92, 49.84, 46.58, 46.53, 46.30, 45.48, 44.34, 44.30, 43.37, 41.26, 41.20, 41.11, 41.04, 35.11, 35.07, 32.28, 30.65, 30.64, $29.88,25.05,22.77$

LCMS (ESI): $m / z[\mathrm{M}+\mathrm{H}]^{+}$calcd for $\left[\mathrm{C}_{17} \mathrm{H}_{25} \mathrm{~N}_{3} \mathrm{O}_{2}\right]^{+}:$304.4; found: 304 .

2-[2-(4-Benzo[b][1,4]benzothiazepin-6-ylpiperazin-1yl)ethoxy]ethanol $(3 \mathbf{r})^{57}$

Yield: $0.5907 \mathrm{~g}$ (91\%); pale-yellow viscous liquid; $R_{f}=0.45$ (hexanes/EtOAc, 30:70).

IR (neat): 2913, 2855, 2361, 2338, 1593, 1574, 1555, 1454, 1404, 1369, 1304, 1242, 1146, 1111, 1061, 1011, 949, 883, 833, 741, 691, $667,617,590,505,463 \mathrm{~cm}^{-1}$.

${ }^{1} \mathrm{H}$ NMR $\left(500 \mathrm{MHz}, \mathrm{CDCl}_{3}\right): \delta=7.49\left(\mathrm{~d}, J=7.6 \mathrm{~Hz}, 1 \mathrm{H}, \mathrm{H}_{\mathrm{Ar}}\right), 7.38(\mathrm{dd}$, $\left.J=7.7,1.3 \mathrm{~Hz}, 1 \mathrm{H}, \mathrm{H}_{\mathrm{Ar}}\right), 7.32-7.27\left(\mathrm{~m}, 3 \mathrm{H}, \mathrm{H}_{\mathrm{Ar}}\right), 7.17-7.14(\mathrm{~m}, 1 \mathrm{H}$, $\mathrm{H}_{\mathrm{Ar}}$ ), $7.07\left(\mathrm{dd}, J=8.0,1.2 \mathrm{~Hz}, 1 \mathrm{H}, \mathrm{H}_{\mathrm{Ar}}\right), 6.87(\mathrm{td}, J=7.6,1.3 \mathrm{~Hz}, 1 \mathrm{H}$, $\left.\mathrm{H}_{\mathrm{Ar}}\right), 3.70-3.57\left(\mathrm{~m}, 10 \mathrm{H}, \mathrm{N}-\mathrm{CH}_{2}\right), 3.57$ (br s, $\left.1 \mathrm{H}, \mathrm{OH}\right), 2.58(\mathrm{dt}, J=14.8$, $\left.6.5 \mathrm{~Hz}, 6 \mathrm{H}, \mathrm{O}-\mathrm{CH}_{2}\right)$.

${ }^{13} \mathrm{C}$ NMR $\left(126 \mathrm{MHz}, \mathrm{CDCl}_{3}\right): \delta=160.68,148.87,139.89,134.06$, $132.21,132.18,130.85,129.13,128.99,128.33,128.00,125.32$, 122.86, 72.46, 67.54, 61.84, 57.97, 53.10.

LC-MS (ESI): $m / z[\mathrm{M}+\mathrm{H}]^{+}$calcd for $\left[\mathrm{C}_{21} \mathrm{H}_{25} \mathrm{~N}_{3} \mathrm{O}_{2} \mathrm{~S}\right]^{+}: 384.51$; found: 384.

Isobutyl 5-(2,5-Dimethylphenoxy)-2,2-dimethylpentanoate (6t) $)^{54}$ Yield: $0.4854 \mathrm{~g}$ (83\%); colorless liquid; $R_{f}=0.77$ (hexanes/EtOAc, 95:5).

IR (neat): 2955, 2870, 2361, 2330, 1759, 1724, 1612, 1582, 1508, $1470,1416,1389,1312,1261,1192,1130,1045,995,941,841,802$, $768,714,667,586,517 \mathrm{~cm}^{-1}$.

${ }^{1} \mathrm{H}$ NMR $\left(500 \mathrm{MHz}, \mathrm{CDCl}_{3}\right): \delta=6.99\left(\mathrm{~d}, J=7.5 \mathrm{~Hz}, 1 \mathrm{H}, \mathrm{H}_{\mathrm{Ar}}\right), 6.65(\mathrm{~d}, J=$ $\left.7.5 \mathrm{~Hz}, 1 \mathrm{H}, \mathrm{H}_{\mathrm{Ar}}\right), 6.60\left(\mathrm{~s}, 1 \mathrm{H}, \mathrm{H}_{\mathrm{Ar}}\right), 3.92-3.88\left(\mathrm{~m}, 2 \mathrm{H}, \mathrm{O}-\mathrm{CH}_{2}\right), 3.85(\mathrm{~d}$, $\left.J=6.5 \mathrm{~Hz}, 2 \mathrm{H}, \mathrm{O}-\mathrm{CH}_{2}\right), 2.30\left(\mathrm{~s}, 3 \mathrm{H}, \mathrm{CH}_{3}\right), 2.17\left(\mathrm{~s}, 3 \mathrm{H}, \mathrm{CH}_{3}\right), 1.93(\mathrm{dp}$, $J=13.3,6.7 \mathrm{~Hz}, 1 \mathrm{H}, \mathrm{CH}), 1.73\left(\mathrm{~s}, 4 \mathrm{H}, \mathrm{CH}_{2}\right), 1.22\left(\mathrm{~s}, 6 \mathrm{H}, \mathrm{CH}_{3}\right), 0.94(\mathrm{~d}$, $\left.J=6.7 \mathrm{~Hz}, 6 \mathrm{H}, \mathrm{CH}_{3}\right)$.

${ }^{13} \mathrm{C}$ NMR $\left(126 \mathrm{MHz}, \mathrm{CDCl}_{3}\right): \delta=177.83,156.98,136.46,130.31$, $123.61,120.69,111.93,70.53,67.97,42.21,37.20,27.82,25.24$ (3C), 21.43, $19.15(2 \mathrm{C}), 15.79$.

GC-MS (EI, $70 \mathrm{eV}$ ): $m / z$ calcd for $\mathrm{C}_{19} \mathrm{H}_{30} \mathrm{O}_{3}$ : 306.44; found: 306 .

\section{Methyl 5-(2,5-Dimethylphenoxy)-2,2-dimethylpentanoate (6u) $)^{58}$} Yield: $0.0531 \mathrm{~g}$ (9\%); colorless liquid; $R_{f}=0.68$ (hexanes/EtOAc, 95:5). IR (neat): 2947, 2866, 2361, 2334, 1732, 1612, 1585, 1508, 1474, 1389, 1312, 1261, 1196, 1153, 1130, 1045, 991, 941, 849, 802, 772, $714,671,586,544,505 \mathrm{~cm}^{-1}$.

${ }^{1} \mathrm{H}$ NMR $\left(500 \mathrm{MHz}, \mathrm{CDCl}_{3}\right): \delta=6.99\left(\mathrm{~d}, J=7.5 \mathrm{~Hz}, 1 \mathrm{H}, \mathrm{H}_{\mathrm{Ar}}\right), 6.65(\mathrm{~d}, J=$ $\left.7.5 \mathrm{~Hz}, 1 \mathrm{H}, \mathrm{H}_{\mathrm{Ar}}\right), 6.60\left(\mathrm{~s}, 1 \mathrm{H}, \mathrm{H}_{\mathrm{Ar}}\right), 3.90\left(\mathrm{t}, J=5.5 \mathrm{~Hz}, 2 \mathrm{H}, \mathrm{O}-\mathrm{CH}_{2}\right), 3.66$ $\left(\mathrm{s}, 3 \mathrm{H}, \mathrm{O}-\mathrm{CH}_{3}\right), 2.30\left(\mathrm{~s}, 3 \mathrm{H}, \mathrm{CH}_{3}\right), 2.17\left(\mathrm{~s}, 3 \mathrm{H}, \mathrm{CH}_{3}\right), 1.76-1.69(\mathrm{~m}, 4 \mathrm{H}$, $\left.\mathrm{CH}_{2}\right), 1.22\left(\mathrm{~s}, 6 \mathrm{H}, \mathrm{CH}_{3}\right)$.

${ }^{13} \mathrm{C}$ NMR $\left(126 \mathrm{MHz}, \mathrm{CDCl}_{3}\right): \delta=178.30,156.93,136.42,130.28$, 123.55, 120.66, 111.89, 67.83, 51.71, 42.09, 37.11, 25.19, 25.18 (2C), 21.40, 15.75.

GC-MS (EI, $70 \mathrm{eV}$ ): $m / z$ calcd for $\mathrm{C}_{16} \mathrm{H}_{24} \mathrm{O}_{3}$ : 264.36; found: 264 . 
5-(2,5-Dimethylphenoxy)-2,2-dimethylpentanoic Acid (7) ${ }^{54}$

Yield: $0.5463 \mathrm{~g}$ (90\%); off-white solid; $\mathrm{mp} 62-65{ }^{\circ} \mathrm{C} ; R_{\mathrm{f}}=0.50$ (EtOAc/ $\mathrm{MeOH}, 90: 10)$.

IR (neat): 2959, 2916, 2870, 2361, 2342, 1759, 1705, 1612, 1582, $1512,1474,1400,1327,1269,1211,1157,1126,1045,995,937,864$, $802,748,586,555 \mathrm{~cm}^{-1}$.

${ }^{1} \mathrm{H}$ NMR $\left(500 \mathrm{MHz}, \mathrm{CDCl}_{3}\right): \delta=6.99\left(\mathrm{~d}, J=7.5 \mathrm{~Hz}, 1 \mathrm{H}, \mathrm{H}_{\mathrm{Ar}}\right), 6.65(\mathrm{~d}, J=$ $\left.7.5 \mathrm{~Hz}, 1 \mathrm{H}, \mathrm{H}_{\mathrm{Ar}}\right), 6.60\left(\mathrm{~s}, 1 \mathrm{H}, \mathrm{H}_{\mathrm{Ar}}\right), 3.92\left(\mathrm{t}, J=6.0 \mathrm{~Hz}, 2 \mathrm{H}, \mathrm{O}-\mathrm{CH}_{2}\right), 2.30$ $\left(\mathrm{s}, 3 \mathrm{H}, \mathrm{CH}_{3}\right), 2.17\left(\mathrm{~s}, 3 \mathrm{H}, \mathrm{CH}_{3}\right), 1.84-1.71\left(\mathrm{~m}, 4 \mathrm{H}, \mathrm{CH}_{2}\right), 1.25(\mathrm{~s}, 6 \mathrm{H}$, $\mathrm{CH}_{3}$ ).

${ }^{13} \mathrm{C}$ NMR $\left(126 \mathrm{MHz}, \mathrm{CDCl}_{3}\right): \delta=184.97,156.98,136.48,130.35$, 123.64, 120.75, 111.98, 67.93, 42.03, 36.91, 25.18 (2C), 25.01, 21.45, 15.81.

LC-MS (ESI): $m / z[\mathrm{M}+\mathrm{H}]^{+}$calcd for $\left[\mathrm{C}_{15} \mathrm{H}_{22} \mathrm{O}_{3}\right]^{+}:$251.33; found: 251 .

\section{Acknowledgment}

The authors thank the Baburaoji Gholap Research Center for support. We are also thankful to the 'Central Instrumental Facility (CIF)' Savitribai Phule Pune University for analytical support.

\section{Supporting Information}

Supporting information for this article is available online at https://doi.org/10.1055/s-0039-1690334.

\section{References}

(1) (a) Lawrence, S. A. Amines: Synthesis Properties and Applications; Cambridge University Press: Cambridge, 2006. (b) Patai, S. Chemistry of the Amino Group.; Wiley Interscience: New York, 1968. (c) Simplício, A. L.; Clancy, J. M.; Gilmer, J. F. Molecules 2008, 13, 519.

(2) Sheldon, R. A.; Van Bekkum, H. Fine Chemicals through Heterogeneous Catalysis; John Wiley \& Sons: New York, 2008.

(3) Elangovan, S.; Neumann, J.; Sortais, J.-B.; Junge, K.; Darcel, C.; Beller, M. Nat. Commun. 2016, 7, 12641.

(4) Harrison, I. R.; Kozlik, A.; McCarthy, J. F.; Palmer, B. H.; Wakerley, S. B.; Watkins, T. I.; Weighton, D. M. Pestic. Sci. 1973, 4, 901.

(5) Wu, L.; Burgess, K. Org. Lett. 2008, 10, 1779.

(6) Travis, A. S. The Chemistry of Anilines; Rappoport, Z., Ed.; John Wiley \& Sons: New York, 2007, 715.

(7) Seayad, A.; Ahmed, M.; Klein, H.; Jackstell, R.; Gross, T.; Beller, M. Science 2002, 297, 1676.

(8) Cazorla, C.; Pfordt, É.; Duclos, M.-C.; Métay, E.; Lemaire, M. Green Chem. 2011, 13, 2482.

(9) Kan, T.; Fukuyama, T. Chem. Commun. 2004, 4, 353.

(10) For a review on the direct alkylation of primary amines with alkyl halides, see: Salvatore, R. N.; Yoon, C. H.; Jung, K. W. Tetrahedron 2001, 57, 7785.

(11) (a) Sorribes, I.; Junge, K.; Beller, M. J. Am. Chem. Soc. 2014, 136, 14314. (b) Volkov, A.; Tinnis, F.; Adolfsson, H. Org. Lett. 2014, 16 , 680. (c) Lampland, N. L.; Hovey, M.; Mukherjee, D.; Sadow, A. D. ACS Catal. 2015, 5, 4219.

(12) (a) Reddy, P. S.; Kanjilal, S.; Sunitha, S.; Prasad, R. B. N. Tetrahedron Lett. 2007, 48, 8807. (b) Byun, E.; Hong, B.; De Castro, K. A.; Lim, M.; Rhee, H. J. Org. Chem. 2007, 72, 9815. (c) Liao, W.; Chen, Y.; Liu, Y.; Duan, H.; Petersen, J. L.; Shi, X. Chem. Commun. 2009,
42, 6436. (d) Nador, F.; Moglie, Y.; Ciolino, A.; Pierini, A.; Dorn, V.; Yus, M.; Alonso, F.; Radivoy, G. Tetrahedron Lett. 2012, 53, 3156. (e) Bogolubsky, A. V.; Moroz, Y. S.; Mykhailiuk, P. K.; Panov, D. M.; Pipko, S. E.; Konovets, A. I.; Tolmachev, A. ACS Comb. Sci. 2014, 16, 375.

(13) (a) Bhat, R. G.; Ghosh, Y.; Chandrasekaran, S. Tetrahedron Lett. 2004, 45, 7983. (b) Zhen, L.; Lin, Y.; Lianghui, L.; Bing, W.; Xuefeng, F. Chem. Commun. 2013, 49, 4214.

(14) (a) Surry, D. A.; Buchwald, S. L. Angew. Chem. Int. Ed. 2008, 47, 6338. (b) Cawley, M. J.; Cloke, F. G. N.; Fitzmaurice, R. J.; Pearson, S. E.; Scott, J. S.; Caddick, S. Org. Biomol. Chem. 2008, 6, 2820. (c) Zhao, X.; Liu, D.; Guo, H.; Liu, Y.; Zhang, W. J. Am. Chem. Soc. 2011, 133, 19354.

(15) (a) Saidi, O.; Blacker, A. J.; Farah, M. M.; Marsden, S. P.; Williams, J. M. J. Angew. Chem. Int. Ed. 2009, 48, 7375; Angew. Chem. 2009, 121, 7511. (b) Tsai, C.-Y.; Sung, R.; Zhuang, B.-R.; Sung, K. Tetrahedron 2010, 66, 6869. (c) Cui, X.; Dai, X.; Deng, Y.; Shi, F. Chem. Eur. J. 2013, 19, 3665. (d) Yan, T.; Feringa, B. L.; Barta, K. Nat. Commun. 2014, 5, 5602. (e) Kolesnikov, P. N.; Yagafarov, N. Z.; Usanov, D. L.; Maleev, V. I.; Chusov, D. Org. Lett. 2015, 17, 173.

(16) (a) Bhattacharyya, S.; Pathak, U.; Mathur, S.; Vishnoi, S.; Jain, R. RSC Adv. 2014, 4, 18229. (b) Gupta, M.; Paul, S.; Gupta, R. Chin. J. Catal. 2014, 35, 444. (c) Hayat, S.; Atta-ur-Rahman, ; Choudhary, M. I.; Khan, K. M.; Schumann, W.; Bayer, E. Tetrahedron 2001, 57, 9951. (d) Gawande, M. B.; Deshpande, S. S.; Satam, J. R.; Jayaram, R. V. Catal. Commun. 2007, 8, 576. (e) BarHaim, G.; Kol, M. Org. Lett. 2004, 6, 3549. (f) Granchi, C.; Capecchi, A.; Del Frate, G.; Martinelli, A.; Macchia, M.; Minutolo, F.; Tuccinardi, T. Molecules 2015, 20, 8772.

(17) (a) Naskar, S.; Bhattacharjee, M. Tetrahedron Lett. 2007, 48, 3367. (b) Fujita, K.; Li, Z.; Ozekib, N.; Yamaguchi, R. Tetrahedron Lett. 2003, 44, 2687. (c) Kawahara, R.; Fujita, K.; Yamaguchi, R. Adv. Synth. Catal. 2011, 353, 1161. (d) Botta, M.; De Angelis, F.; Nicoletti, R. Synthesis 1977, 722. (e) Tayade, K. N.; Mishra, M.; Munusamy, K.; Somani, R. S. J. Mol. Catal. A: Chem. 2014, 390, 91. (f) Nagaraju, N.; Kuriakose, G. New J. Chem. 2003, 27, 765.

(18) Selva, M.; Tundo, P.; Perosa, A. J. Org. Chem. 2003, 68, 7374.

(19) Llabres-Campaner, P. J.; Ballesteros-Garrido, R.; Ballesteros, R.; Abarca, B. Tetrahedron 2017, 73, 5552.

(20) (a) Freifelder, M.; Stone, G. R. J. Org. Chem. 1961, 26, 1477. (b) Azizi, N.; Saidi, M. R. Org. Lett. 2005, 7, 3649.

(21) (a) Yin, J.; Ye, G.; Wang, X. J. Mater. Chem. C 2013, 1, 3794. (b) Ross, W. C. J.J. Chem. Soc. 1949, 183. (c) Chen, J.; Peng, Z.; Lu, M.; Xiong, X.; Chen, Z.; Li, Q.; Cheng, Z.; Jiang, D.; Tao, L.; Hua, G. Bioorg. Med. Chem. Lett. 2018, 28, 222. (d) Campbell, D.; Dix, L. R.; Rostron, P. Dyes Pigm. 1995, 29, 77. (e) Guo, H.; Zhuang, Y.; Cao, J.; Zhang, G. Synth. Commun. 2014, 44, 3368. (f) Rindfusz, R. E.; Harnack, V. L. J. Am. Chem. Soc. 1920, 42, 1720.

(22) Li, X.-D.; Xia, S.-M.; Chen, K.-H.; Liu, X.-F.; Li, H.-R.; He, L.-N. Green Chem. 2018, 20, 4853.

(23) Brielles, C.; Harnett, J. J.; Dorisa, E. Tetrahedron Lett. 2001, 42, 8301.

(24) (a) Poirot, M.; De Medina, P.; Delarue, F.; Perie, J.-J.; Klaebe, A.; Faye, J.-C. Bioorg. Med. Chem. 2000, 8, 2007. (b) Gupta, P. P.; Sharma, J. N. J. Med. Chem. 1973, 16, 797. (c) Srivastava, S. K.; Chauhan, P. M. S.; Bhaduri, A. P. Synth. Commun. 1999, 29, 2085.

(25) Singh, C. B.; Kavala, V.; Samal, A. K.; Patel, B. K. Eur. J. Org. Chem. 2007, 1369 .

(26) Depreux, P.; Aichaoui, H.; Lesieur, I. Heterocycles 1993, 36, 1051.

(27) (a) Salvatore, R. N.; Nagle, A. S.; Jung, K. W. J. Org. Chem. 2002, 67, 674. (b) Salvatore, R. N.; Nagle, A. S.; Schmidt, S. E.; Jung, K. W. Org. Lett. 1999, 1, 1893. 
(28) (a) Díaz, J. E.; Bisceglia, J. Á.; Mollo, M. C.; Orelli, L. R. Tetrahedron Lett. 2011, 52, 1895. (b) Fink, D. M. Synlett 2004, 2394. (c) Castillo, J.-C.; Orrego-Hernández, J.; Portilla, J. Eur. J. Org. Chem. 2016, 3824.

(29) (a) Monopoli, A.; Cotugno, P.; Cortese, M.; Calvano, C. D.; Ciminale, F.; Nacci, A. Eur. J. Org. Chem. 2012, 3105. (b) Chiappe, C.; Piccioli, P.; Pieraccini, D. Green Chem. 2006, 8, 277.

(30) Cardullo, F.; Donati, D.; Fusillo, V.; Merlo, G.; Paio, A.; Salaris, M.; Solinas, A.; Taddei, M. J. Comb. Chem. 2006, 8, 834.

(31) Landge, V. G.; Mondal, A.; Kumar, V.; Nandakumar, A.; Balaraman, E. Org. Biomol. Chem. 2018, 16, 8175.

(32) Vellakkaran, M.; Singh, K.; Banerjee, D. ACS Catal. 2017, 7, 8152.

(33) Ogata, O.; Nara, H.; Fujiwhara, M.; Matsumura, K.; Kayaki, Y. Org. Lett. 2018, 20, 3866.

(34) Basu, B.; Paul, S.; Nanda, A. K. Green Chem. 2009, 11, 1115.

(35) (a) Williamson, A. W. J. Chem. Soc. 1852, 229. (b) Fuhrmann, E.; Talbiersky, J. Org. Process Res. Dev. 2005, 9, 206. (c) Mandal, S.; Mandal, S.; Ghosh, S. K.; Sar, P.; Ghosh, A.; Saha, R.; Saha, B. RSC Adv. 2016, 6, 69605.

(36) Sueki, S.; Kuninobu, Y. Org. Lett. 2013, 15, 1544.

(37) (a) Ando, T.; Yamawaki, J.; Kawate, T.; Sumi, S.; Hanafusa, T. Bull. Chem. Soc.Jpn. 1982, 55, 2504. (b) Xu, W.; Mohan, R.; Morrissey, M. M. Tetrahedron Lett. 1997, 38, 7337. (c) Huston, R. C.; Guile, R. L.; Chen, P. S.; Headley, W. N.; Warren, G. W.; Baur, L. S.; Mate, B. O. J. Am. Chem. Soc. 1933, 55, 4639. (d) Johnstone, R. A. W.; Rose, M. E. Tetrahedron 1979, 35, 2169. (e) Keglevich, G.; Bálint, E.; Karsai, É.; Grün, A.; Bálint, M.; Greiner, I. Tetrahedron Lett. 2008, 49, 5039. (f) De Zani, D.; Colombo, M. J. Flow Chem. 2012, 2, 5. (g) Bogdal, D.; Pielichowski, J.; Boron, A. Synth. Commun. 1998, 28, 3029. (h) Brieger, G.; Hachey, D.; Nestrick, T. J. Chem. Eng. Data 1968, 13, 581. (i) Bu, X.; Jing, H.; Wang, L.; Chang, T.; Jin, L.; Liang, Y. J. Mol. Catal. A: Chem. 2006, 259, 121.

(38) (a) Cazorla, C.; Pfordt, E.; Duclos, M.-C.; Métay, E.; Lemaire, M. Green Chem. 2011, 13, 2482. (b) Lindstedt, E.; Ghosh, R.; Olofsson, B. Org. Lett. 2013, 15, 6070. (c) Samolada, M. C.; Grigoriadou, E.; Kiparissides, Z.; Vasalos, I. A. J. Catal. 1995, 152, 52.

(39) (a) Basak, A.; Nayak, M. K.; Chakraborti, A. K. Tetrahedron Lett. 1998, 39, 4883. (b) Perosa, A.; Selva, M.; Tundo, P.; Zordan, F. Synlett 2000, 272.

(40) Trost, B. M.; Toste, F. D. J. Am. Chem. Soc. 1998, 120, 815.

(41) (a) Teruo, Y.; Shigeru, I.; Yoshiharu, I. Bull. Chem. Soc. Jpn. 1973, 46, 553. (b) Wang, S.; Dupin, L.; Noël, M.; Carroux, C. J.; Renaud, L.; Géhin, T.; Meyer, A.; Souteyrand, E.; Vasseur, J.-J.; Vergoten, G.; Chevolot, Y.; Morvan, F.; Vidal, S. Chem. Eur. J. 2016, 22, 11785 .
(42) Yamansarova, E. T.; Kukovinets, A. G.; Kukovinets, O. S.; Zainullin, R. A.; Galin, F. Z.; Kunakova, R. V.; Zorin, V. V.; Tolstikov, G. A. Russ. J. Org. Chem. 2001, 37, 246.

(43) (a) Turgut, Y.; Aral, T.; Karakaplan, M.; Deniz, P.; Hosgoren, H. Synth. Commun. 2010, 40, 3365. (b) Rastogi, S. N.; Anand, N.; Gupta, P. P.; Sharma, J. N. J. Med. Chem. 1973, 16, 797.

(44) (a) Purushothaman, S.; Prasanna, R.; Niranjana, P.; Raghunathan, R.; Nagaraj, S.; Rengasamy, R. Bioorg. Med. Chem. Lett. 2010, 20, 7288. (b) Cho, W. S.; Kim, S. H.; Kim, D. J.; Mun, S.-D.; Kim, R.; Go, M. J.; Park, M. H.; Kim, M.; Lee, J.; Kim, Y. Polyhedron 2014, 67, 205. (c) Dong, M.; Si, Y. Q.; Sun, S. Y.; Pu, X. P.; Yang, Z. J.; Zhang, L. R.; Zhang, L. H.; Leung, F. P.; Lam, C. M. C.; Kwong, A. K. Y.; Yue, J. Org. Biomol. Chem. 2011, 9, 3246.

(45) Morales, P.; Gomez-Canas, M.; Navarro, G.; Hurst, D. P.; CarrilloSalinas, F. J.; Lagartera, L.; Pazos, R.; Goya, P.; Reggio, P. H.; Guaza, C.; Franco, R.; Fernandez-Ruiz, J.; Jagerovic, N. J. Med. Chem. 2016, 59, 6753.

(46) Hu, Z.; Zhang, S.; Zhou, W.; Ma, X.; Xiang, G. Bioorg. Med. Chem. Lett. 2017, 27, 1854.

(47) Parrish, J. P.; Sudaresan, B.; Jung, K. W. Synth. Commun. 1999, 29, 4423.

(48) More, S. V.; Ardhapure, S. S.; Naik, N. H.; Bhusare, S. R.; Jadhav, W. N.; Pawar, R. P. Synth. Commun. 2005, 35, 3113.

(49) (a) Dermer, O. C. Chem. Rev. 1934, 14, 385. (b) Mazaleyrat, J.-P.; Wakselman, M. J. Org. Chem. 1996, 61, 2695.

(50) (a) Platonov, A. Y.; Evdokimov, A. N.; Kurzin, A. V.; Maiyorova, H. D. J. Chem. Eng. Data 2002, 47, 1175. (b) Stenger, V. A. J. Chem. Eng. Data 1996, 41, 1111.

(51) For ticlopidine synthesis: (a) Maffrand, J. P.; Eloy, F. Eur. J. Med. Chem. 1974, 9, 483. (b) Maffrand, J. P.; Eloy, F. J. Heterocycl. Chem. 1976, 13, 1347.

(52) For vildagliptin synthesis: (a) Deng, Y.; Wang, A.; Tao, Z.; Chen, Y.; Pan, X.; Hu, X. Lett. Org. Chem. 2014, 11, 780. (b) Castaldi, M.; Baratella, M.; Menegotto, I. G.; Castaldi, G.; Giovenzana, G. B. Tetrahedron Lett. 2017, 58, 3426.

(53) For quetiapine synthesis: Bharathi, C. H.; Prabahar, K. J.; Prasad, C. S.; Srinivasa Rao, M.; Trinadhachary, G. N.; Handa, V. K.; Dandala, R.; Naidu, A. Pharmazie 2008, 63, 14.

(54) For gemfibrozil synthesis: (a) Nunna, R.; Jayanna, N. D.; Ramachandran, D. Asian J. Chem. 2015, 27, 925. (b) Madasu, S. B.; Vekariya, N. A.; Velladurai, H.; Islam, A.; Sanasi, P. D.; Korupolu, R. B. Org. Process Res. Dev. 2013, 17, 963.

(55) Aillaud, I.; Haurena, C.; Gall, E. L.; Martens, T.; Ricci, G. Molecules 2010, $15,8144$.

(56) Xu, X.; Guo, J.; Su, Q.; Zhong, X. Asian J. Chem. 2013, 25, 7557.

(57) Li, M.; Wang, J. J. Org. Lett. 2018, 20, 6490.

(58) McManus, J. B.; Nicewicz, D. A. J. Am. Chem. Soc. 2017, 139, 2880. 